\title{
Improved Stabilization Conditions for Nonlinear Systems with Input and State Delays via T-S Fuzzy Model
}

\author{
Chang Che, ${ }^{1,2}$ Jiayao Peng, ${ }^{1}$ Tao Zhao, ${ }^{3}$ Jian Xiao, ${ }^{2}$ and Jie Zhou ${ }^{4}$ \\ ${ }^{1}$ School of Mechanical Engineering, Xihua University, Chengdu 610039, China \\ ${ }^{2}$ School of Electrical Engineering, Southwest Jiaotong University, Chengdu 610031, China \\ ${ }^{3}$ School of Electrical Engineering and Information, Sichuan University, Chengdu 610065, China \\ ${ }^{4}$ School of Computer and Software Engineering, Xihua University, Chengdu 610039, China \\ Correspondence should be addressed to Jie Zhou; zibing357@163.com
}

Received 11 October 2017; Revised 23 February 2018; Accepted 12 March 2018; Published 16 April 2018

Academic Editor: Xuejun Xie

Copyright (c) 2018 Chang Che et al. This is an open access article distributed under the Creative Commons Attribution License, which permits unrestricted use, distribution, and reproduction in any medium, provided the original work is properly cited.

\begin{abstract}
This paper focuses on the problem of nonlinear systems with input and state delays. The considered nonlinear systems are represented by Takagi-Sugeno (T-S) fuzzy model. A new state feedback control approach is introduced for T-S fuzzy systems with input delay and state delays. A new Lyapunov-Krasovskii functional is employed to derive less conservative stability conditions by incorporating a recently developed Wirtinger-based integral inequality. Based on the Lyapunov stability criterion, a series of linear matrix inequalities (LMIs) are obtained by using the slack variables and integral inequality, which guarantees the asymptotic stability of the closed-loop system. Several numerical examples are given to show the advantages of the proposed results.
\end{abstract}

\section{Introduction}

In recent years, T-S fuzzy system has become a very active research direction in the fields of fuzzy control [1-8]. T-S fuzzy model is useful for approximating complex nonlinear systems by local linear submodel and fuzzy membership functions. It has been proved that T-S fuzzy model can approximate any smooth nonlinear dynamic systems. Therefore, T-S fuzzy model is a very effective and powerful tool for the study of nonlinear systems. Currently, many valuable results on stability analysis, controller design, filter design, and fault detection of T-S fuzzy systems have been widely reported in [9-19].

It is well known that time-delay is an important source of system instability or oscillation [20-27]. LyapunovKrasovskii functional (LKF) technique is an effective method to handle stability analysis and controller design of T-S fuzzy time-delay system. Various methods are introduced to obtain less conservative stability conditions for T-S fuzzy timedelay system. There are two main relaxed techniques for T$S$ fuzzy time-delay system, for example, constructing new LKF with more system information and using new bounding inequalities. A variety of results have been presented for TS fuzzy time-delay system by combining LKF and bounding inequality approaches [28-37]. However, the existing results are still very conservative due to the form of LKF, and thus constructing new LKF with more system information is a challenging issue to reduce the conservativeness of stability analysis for T-S fuzzy time-delay system.

The aforementioned results only consider the state delays in T-S fuzzy systems. Therefore, these approaches mentioned above may be invalid when they are used to control the systems with input delays. It is well known that input delays always occur in practical control systems. Therefore, it is very significant to study stability and controller design for T-S fuzzy systems with state and input delays. Therefore, a few studies have been developed for T-S fuzzy systems with state and input delays [38-40]. However, the existing results are very conservative because LFK including double integral terms are only used to derive stability conditions in the form of LMIs.

In this paper, the stabilization problem of nonlinear systems with input and state delays is studied by using T$S$ fuzzy model. Novel stabilization conditions are presented 
by Lyapunov stability theory. The main contributions of this paper can be summarized as follows. (1) A new LKF with triple integral terms is for the first time constructed for T-S fuzzy model with input and state delays. (2) Wirtinger-based double integral inequality is employed to derive LMI-based stabilization conditions. (3) Using LKF with more system information, Wirtinger-based double integral inequality, and matrix transformation technique, less conservative stabilization conditions are proposed for nonlinear systems with input and state delays. The paper is organized as follows. The problem descriptions and some useful lemmas are presented in Section 2. The main derivation process is given in Section 3. Several numerical examples are provided to demonstrate the effectiveness of the proposed approach in Section 4. The conclusion is drawn in the last section.

\section{Preliminaries}

2.1. Problem Description. Consider a nonlinear system described by T-S fuzzy model with input and state delays. The rules are defined as follows.

Plant Rule $i$. If $\theta_{1}(t)$ is $M_{i 1}$ and $\theta_{2}(t)$ is $M_{i 2}$ and $\ldots$ and $\theta_{p}(t)$ is $M_{i p}$, then

$$
\begin{aligned}
& \dot{x}(t)=A_{i} x(t)+A_{d i} x(t-h)+B_{i} u(t-\tau), \\
& x(t)=\phi(t), \quad t \in[-\max (h, \tau), 0],
\end{aligned}
$$

$$
i=1,2, \ldots, r \text {, }
$$

where $x(t)=\left[x_{1}(t), x_{2}(t), \ldots, x_{n}(t)\right]^{\mathrm{T}} \in R^{n}$ is the state; $\phi(t)$ is the initial condition defined on $t \in[-\max (h, \tau), 0] ; M_{i j}$ is the fuzzy set; $r$ is the number of plant rules; and $\theta(t)=$ $\left[\theta_{1}(t), \theta_{2}(t), \ldots, \theta_{p}(t)\right]$ is the antecedent variable vector. $h$ and $\tau$, respectively, indicate the state delay and input delay. $A_{i}, B_{i}$, and $A_{d i}$ are known real constant matrices with appropriate dimensions.

The overall fuzzy model can be given by

$$
\begin{aligned}
\dot{x}(t) & \\
& =\sum_{i=1}^{r} \lambda_{i}(\theta(t))\left[A_{i} x(t)+A_{d i} x(t-h)+B_{i} u(t-\tau)\right],
\end{aligned}
$$

where

$$
\begin{aligned}
\sum_{i=1}^{r} \lambda_{i}(\theta(t)) & =1, \\
\lambda_{i}(\theta(t)) & =\frac{\omega_{i}(\theta(t))}{\sum_{i=1}^{r} \omega_{i}(\theta(t))}, \\
\omega_{i}(\theta(t)) & =\prod_{j=1}^{r} M_{i j}\left(\theta_{j}(t)\right),
\end{aligned}
$$

and $M_{i j}\left(\theta_{j}(t)\right)$ indicates the membership degree of $\theta_{j}(t)$ in $M_{i j}$.

A tight T-S fuzzy model can be written as

$$
\dot{x}(t)=A(\lambda) x(t)+A_{d}(\lambda) x(t-h)+B(\lambda) u(t-\tau),
$$

$$
x(t)=\phi(t), \quad t \in[-\max (h, \tau), 0],
$$

where $A(\lambda)=\sum_{i=1}^{r} \lambda_{i}(\theta(t)) A_{i}, A_{d}(\lambda)=\sum_{i=1}^{r} \lambda_{i}(\theta(t)) A_{d i}$, and $B(\lambda)=\sum_{i=1}^{r} \lambda_{i}(\theta(t)) B_{i}$.

Moreover, we define the fuzzy state feedback control rules using the following fuzzy parallel distributed compensation strategy.

Controller Rule $i$. If $\theta_{1}(t)$ is $M_{i 1}$ and $\theta_{2}(t)$ is $M_{i 2}$ and ... and $\theta_{p}(t)$ is $M_{i p}$, then

$$
u(t)=K_{i} x(t), \quad i=1,2, \ldots, r,
$$

where $x(t)$ is the input, $u(t) \in R^{m}$ is the output, and $K_{i}$ is the gain matrix of the controller.

So the overall output of the fuzzy state feedback controller can be represented as

$$
u(t)=\sum_{i=1}^{r} \lambda_{i}(\theta(t)) K_{i} x(t) .
$$

The compact form can be written as

$$
u(t)=K(\lambda) x(t)
$$

where $K(\lambda)=\sum_{i=1}^{r} \lambda_{i}(\theta(t)) K_{i}$.

Therefore, we can get the closed-loop system formed by T-S fuzzy system with input delay and state delays in (2) and state feedback controller in (6), which is as follows:

$$
\begin{aligned}
\dot{x}(t)= & \sum_{i=1}^{r} \sum_{j=1}^{r} \lambda_{i}(\theta(t)) \lambda_{j}(\theta(t)) \\
& \cdot\left[A_{i} x(t)+A_{d i} x(t-h)+B_{i} K_{j} x(t-\tau)\right] .
\end{aligned}
$$

The compact form of the closed-loop system is represented as

$$
\begin{aligned}
\dot{x}(t)= & A(\lambda) x(t)+A_{d}(\lambda) x(t-h) \\
& +B(\lambda) K(\lambda) x(t-\tau) .
\end{aligned}
$$

The goal of this paper is to find gains $K_{i}$ such that the closed-loop system (9) is asymptotically stable.

2.2. Useful Lemmas. The following lemmas are useful to obtain the main results of this paper.

Lemma 1 (see [25]). For any constant matrix $M>0$, given scalars $a$ and $b$ satisfying $a<b$, the following inequality holds for all continuously differentiable function $\varphi$ in $[a, b] \rightarrow R^{n}$ :

$$
\begin{aligned}
(b & -a) \int_{a}^{b} \varphi^{\mathrm{T}}(s) M \varphi(s) d s \\
& \geq\left(\int_{a}^{b} \varphi(s) d s\right)^{\mathrm{T}} M\left(\int_{a}^{b} \varphi(s) d s\right)+3 \Theta_{d}^{\mathrm{T}} M \Theta_{d}
\end{aligned}
$$

where $\Theta_{d}=\int_{a}^{b} \varphi(s) d s-(2 /(b-a)) \int_{a}^{b} \int_{a}^{s} \varphi(u) d u d s=$ $-\int_{a}^{b} \varphi(s) d s+(2 /(b-a)) \int_{a}^{b} \int_{s}^{b} \varphi(u) d u d s$. 
Lemma 2 (see [26]). For a given matrix $M>0$, given scalars $a$ and $b$ satisfying $a<b$, the following inequality holds for all continuously differentiable function $x$ in $[a, b] \rightarrow R^{n}$ :

$$
\begin{aligned}
& \frac{(b-a)^{2}}{2} \int_{a}^{b} \int_{s}^{b} x^{\mathrm{T}}(u) M x(u) d u d s \\
& \geq\left(\int_{a}^{b} \int_{s}^{b} x(u) d u d s\right)^{\mathrm{T}} M\left(\int_{a}^{b} \int_{s}^{b} x(u) d u d s\right) \\
& \quad+2 \Theta_{d}^{\mathrm{T}} M \Theta_{d},
\end{aligned}
$$

where $\Theta_{d}=-\int_{a}^{b} \int_{s}^{b} x(u) d u d s+(3 /(b-$ a)) $\int_{a}^{b} \int_{s}^{b} \int_{u}^{b} x(v) d v d u d s$

Lemma 3 (see [41]). Given matrices $v \in R^{n}, \Theta=\Theta^{\mathrm{T}} \in R^{n \times n}$, and $N \in R^{m \times n}$, if $\operatorname{rank}(N)<n$, then

$$
v^{\mathrm{T}} \Theta v<0, \quad \forall N v=0, \quad v \neq 0
$$

if and only if there exists matrix $L \in R^{n \times m}$ such that

$$
\Theta+L N+N^{\mathrm{T}} L^{\mathrm{T}}<0 .
$$

\section{Main Results}

In this section, new stability conditions for system (9) will be presented. Now we give the following theorem.

Theorem 4. Consider the closed-loop system (9) and given scalars $h$ and $\tau$ to meet $h>0, \tau>0$, the system is asymptotically stable, if there exist scalars $a_{1}$ and $a_{2}$, matrices $M(\lambda), X$, and positive definite symmetric matrices $\widehat{P}, \widehat{Q}, \widehat{D}, \widehat{T}$, $\widehat{\bar{Q}}, \widehat{\bar{D}}$, and $\widehat{\bar{T}}$, such that the following inequality holds:

$$
\widehat{\Xi}+\widehat{L} \widehat{\Gamma}(\lambda)+\widehat{\Gamma}(\lambda)^{\mathrm{T}} \widehat{L}^{\mathrm{T}}<0,
$$

where

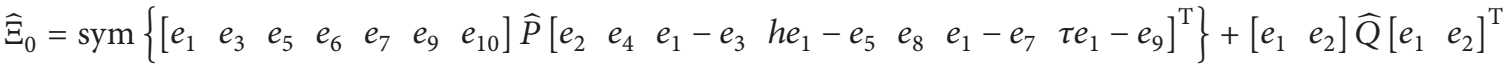

$$
\begin{aligned}
& -\left[\begin{array}{ll}
e_{3} & e_{4}
\end{array}\right] \widehat{\mathrm{Q}}\left[\begin{array}{ll}
e_{3} & e_{4}
\end{array}\right]^{\mathrm{T}}+h^{2}\left[\begin{array}{ll}
e_{1} & e_{2}
\end{array}\right] \widehat{D}\left[\begin{array}{ll}
e_{1} & e_{2}
\end{array}\right]^{\mathrm{T}}+\left(\frac{h^{2}}{2}\right)^{2} e_{2} \widehat{T} e_{2}{ }^{\mathrm{T}}+\left[\begin{array}{ll}
e_{1} & e_{2}
\end{array}\right] \widehat{\bar{Q}}\left[\begin{array}{ll}
e_{1} & e_{2}
\end{array}\right]^{\mathrm{T}}-\left[\begin{array}{ll}
e_{7} & e_{8}
\end{array}\right] \widehat{\bar{Q}}\left[e_{7} e_{8}\right]^{\mathrm{T}} \\
& +\tau^{2}\left[\begin{array}{ll}
e_{1} & e_{2}
\end{array}\right] \widehat{\bar{D}}\left[\begin{array}{ll}
e_{1} & e_{2}
\end{array}\right]^{\mathrm{T}}+\left(\frac{\tau^{2}}{2}\right)^{2} e_{2} \widehat{\bar{T}} e_{2}{ }^{\mathrm{T}} \\
& \widehat{\Xi}_{1}=-\left[\begin{array}{ll}
e_{5} & e_{1}-e_{3}
\end{array}\right] \widehat{D}\left[\begin{array}{ll}
e_{5} & e_{1}-e_{3}
\end{array}\right]^{\mathrm{T}}-3\left[\left(\frac{2}{h}\right) e_{6}-e_{5} e_{1}+e_{3}-\left(\frac{2}{h}\right) e_{5}\right] \widehat{D}\left[\left(\frac{2}{h}\right) e_{6}-e_{5} e_{1}+e_{3}-\left(\frac{2}{h}\right) e_{5}\right]^{\mathrm{T}} \text {, } \\
& \widehat{\Xi}_{2,1}=-\left[h e_{1}-e_{5}\right] \widehat{T}\left[h e_{1}-e_{5}\right]^{\mathrm{T}}, \\
& \widehat{\Xi}_{2,2}=-2\left[\left(\frac{h}{2}\right) e_{1}+e_{5}-\left(\frac{3}{h}\right) e_{6}\right] \widehat{T}\left[\left(\frac{h}{2}\right) e_{1}+e_{5}-\left(\frac{3}{h}\right) e_{6}\right]^{\mathrm{T}} \text {, } \\
& \widehat{\Xi}_{3}=-\left[\begin{array}{ll}
e_{9} & e_{1}-e_{7}
\end{array}\right] \widehat{\bar{D}}\left[\begin{array}{ll}
e_{9} & e_{1}-e_{7}
\end{array}\right]^{\mathrm{T}}-3\left[\left(\frac{2}{\tau}\right) e_{10}-e_{9} e_{1}+e_{7}-\left(\frac{2}{\tau}\right) e_{9}\right] \widehat{\bar{D}}\left[\left(\frac{2}{\tau}\right) e_{10}-e_{9} e_{1}+e_{7}-\left(\frac{2}{\tau}\right) e_{9}\right]^{\mathrm{T}} \text {, } \\
& \widehat{\Xi}_{4,1}=-\left[\tau e_{1}-e_{9}\right] \widehat{\bar{T}}\left[\tau e_{1}-e_{9}\right]^{\mathrm{T}}, \\
& \widehat{\Xi}_{4,2}=-2\left[\left(\frac{\tau}{2}\right) e_{1}+e_{9}-\left(\frac{3}{\tau}\right) e_{10}\right] \widehat{\bar{T}}\left[\left(\frac{\tau}{2}\right) e_{1}+e_{9}-\left(\frac{3}{\tau}\right) e_{10}\right]^{\mathrm{T}}, \\
& \widehat{\Xi}=\widehat{\Xi}_{0}+\widehat{\Xi}_{1}+\widehat{\Xi}_{2,1}+\widehat{\Xi}_{2,2}+\widehat{\Xi}_{3}+\widehat{\Xi}_{4,1}+\widehat{\Xi}_{4,2} \text {, } \\
& \widehat{L}=a_{1} e_{1}+e_{2}+a_{2} e_{3} \text {, } \\
& \widehat{\Gamma}(\lambda)=\left[\begin{array}{llll}
A(\lambda) X & -X & A_{d}(\lambda) X & B(\lambda) M(\lambda)
\end{array}\right]\left[e_{1}, e_{2}, e_{3}, e_{7}\right]^{\mathrm{T}} .
\end{aligned}
$$

In addition, the gain matrix of state feedback controller can be obtained as

$$
K(\lambda)=M(\lambda) X^{-1} .
$$

Proof. In order to establish a stability condition of system (9), we choose the following Lyapunov-Krasovskii functional:

$$
\begin{gathered}
V=v_{1}^{\mathrm{T}}(t) P v_{1}(t)+\int_{t-h}^{t} v_{2}^{\mathrm{T}}(s) Q v_{2}(s) d s \\
+h \int_{t-h}^{t} \int_{s}^{t} v_{2}^{\mathrm{T}}(u) D v_{2}(u) d u d s
\end{gathered}
$$




$$
\begin{aligned}
& +\frac{h^{2}}{2} \int_{t-h}^{t} \int_{s}^{t} \int_{u}^{t} \dot{x}^{\mathrm{T}}(v) T \dot{x}(v) d v d u d s \\
& +\int_{t-\tau}^{t} v_{2}^{\mathrm{T}}(s) \bar{Q} v_{2}(s) d s \\
& +\tau \int_{t-\tau}^{t} \int_{s}^{t} v_{2}^{\mathrm{T}}(u) \bar{D} v_{2}(u) d u d s \\
& +\frac{\tau^{2}}{2} \int_{t-\tau}^{t} \int_{s}^{t} \int_{u}^{t} \dot{x}^{\mathrm{T}}(v) \bar{T} \dot{x}(v) d v d u d s,
\end{aligned}
$$

where

$$
\begin{aligned}
v_{1}(t) & =\left[\begin{array}{c}
x(t) \\
x(t-h) \\
\int_{t-h}^{t} x(s) d s \\
\int_{t-h}^{t} \int_{s}^{t} x(u) d u d s \\
x(t-\tau) \\
v_{2}^{t}(t)=\left[\begin{array}{c}
\int_{t-\tau}^{t}(s) d s \\
\int_{t-\tau}^{t} x(t) \\
\dot{x}(t)
\end{array}\right], \\
\dot{v}_{1}(t)= \\
\dot{x}(t) \\
\dot{x}(t-h) \\
x(t)-x(t-h) \\
h x(t)-\int_{t-h}^{t} x(s) d s \\
\dot{x}(t-\tau) \\
x(t)-x(t-\tau) \\
\tau x(t)-\int_{t-\tau}^{t} x(s) d s
\end{array}\right] .
\end{aligned}
$$

$$
\begin{aligned}
& \xi_{1}(t)=\int_{t-h}^{t} v_{2}^{\mathrm{T}}(s) D v_{2}(s) d s, \\
& \xi_{2}(t)=\int_{t-h}^{t} \int_{s}^{t} \dot{x}^{\mathrm{T}}(u) T \dot{x}(u) d u d s, \\
& \xi_{3}(t)=\int_{t-\tau}^{t} v_{2}^{\mathrm{T}}(s) \bar{D} v_{2}(s) d s \\
& \xi_{4}(t)=\int_{t-\tau}^{t} \int_{s}^{t} \dot{x}^{\mathrm{T}}(u) \bar{T} \dot{x}(u) d u d s
\end{aligned}
$$

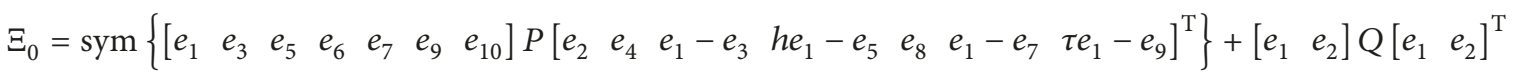$$
-\left[\begin{array}{ll}
e_{3} & e_{4}
\end{array}\right] Q\left[\begin{array}{ll}
e_{3} & e_{4}
\end{array}\right]^{\mathrm{T}}+h^{2}\left[\begin{array}{ll}
e_{1} & e_{2}
\end{array}\right] D\left[e_{1} e_{2}\right]^{\mathrm{T}}+\left(\frac{h^{2}}{2}\right)^{2} e_{2} T e_{2}^{\mathrm{T}}+\left[\begin{array}{ll}
e_{1} & e_{2}
\end{array}\right] \bar{Q}\left[\begin{array}{ll}
e_{1} & e_{2}
\end{array}\right]^{\mathrm{T}}-\left[\begin{array}{ll}
e_{7} & e_{8}
\end{array}\right] \bar{Q}\left[\begin{array}{ll}
e_{7} & e_{8}
\end{array}\right]^{\mathrm{T}}
$$

Moreover, $P \in R^{7 n \times 7 n}, Q, \bar{Q} \in R^{2 n \times 2 n}, D, \bar{D} \in R^{2 n \times 2 n}$, and $T, \bar{T} \in R^{n \times n}$ are positive definite symmetric matrices.

The time-derivative of $V$ can be computed as

$$
\begin{aligned}
& \dot{V}=2 v_{1}^{\mathrm{T}}(t) P \dot{v}_{1}(t)+v_{2}^{\mathrm{T}}(t) Q v_{2}(t) \\
& -v_{2}^{\mathrm{T}}(t-h) Q v_{2}(t-h)+h^{2} v_{2}^{\mathrm{T}}(t) D v_{2}(t) \\
& -h \xi_{1}(t)+\left(\frac{h^{2}}{2}\right)^{2} \dot{x}^{\mathrm{T}}(t) T \dot{x}(t)-\frac{h^{2}}{2} \xi_{2}(t) \\
& +v_{2}^{\mathrm{T}}(t) \overline{\mathrm{Q}} v_{2}(t)-v_{2}^{\mathrm{T}}(t-\tau) \overline{\mathrm{Q}} v_{2}(t-\tau) \\
& +\tau^{2} v_{2}^{\mathrm{T}}(t) \bar{D} v_{2}(t)-\tau \xi_{3}(t) \\
& +\left(\frac{\tau^{2}}{2}\right)^{2} \dot{x}^{\mathrm{T}}(t) \bar{T} \dot{x}(t)-\frac{\tau^{2}}{2} \xi_{4}(t) \\
& =2 v_{1}^{\mathrm{T}}(t) P \dot{v}_{1}(t)+v_{2}^{\mathrm{T}}(t) \mathrm{Q} v_{2}(t) \\
& -v_{2}^{\mathrm{T}}(t-h) \mathrm{Q} v_{2}(t-h)+h^{2} v_{2}^{\mathrm{T}}(t) D v_{2}(t) \\
& +\left(\frac{h^{2}}{2}\right)^{2} \dot{x}^{\mathrm{T}}(t) T \dot{x}(t)+v_{2}^{\mathrm{T}}(t) \bar{Q} v_{2}(t) \\
& -v_{2}^{\mathrm{T}}(t-\tau) \bar{Q} v_{2}(t-\tau)+\tau^{2} v_{2}^{\mathrm{T}}(t) \bar{D} v_{2}(t) \\
& +\left(\frac{\tau^{2}}{2}\right)^{2} \dot{x}^{\mathrm{T}}(t) \bar{T} \dot{x}(t)-h \xi_{1}(t)-\frac{h^{2}}{2} \xi_{2}(t) \\
& -\tau \xi_{3}(t)-\frac{\tau^{2}}{2} \xi_{4}(t) \\
& =\zeta^{\mathrm{T}}(t) \Xi_{0} \zeta(t)-h \xi_{1}(t)-\frac{h^{2}}{2} \xi_{2}(t)-\tau \xi_{3}(t) \\
& -\frac{\tau^{2}}{2} \xi_{4}(t)
\end{aligned}
$$

where 
Mathematical Problems in Engineering

5

$$
\begin{aligned}
& +\tau^{2}\left[\begin{array}{ll}
e_{1} & e_{2}
\end{array}\right] \bar{D}\left[\begin{array}{ll}
e_{1} & e_{2}
\end{array}\right]^{\mathrm{T}}+\left(\frac{\tau^{2}}{2}\right)^{2} e_{2} \bar{T} e_{2}{ }^{\mathrm{T}} \\
& \zeta^{\mathrm{T}}(t)=\left[x^{\mathrm{T}}(t), \dot{x}^{\mathrm{T}}(t), x^{\mathrm{T}}(t-h), \dot{x}^{\mathrm{T}}(t-h), \int_{t-h}^{t} x^{\mathrm{T}}(s) d s, \int_{t-h}^{t} \int_{s}^{t} x^{\mathrm{T}}(u) d u d s, x^{\mathrm{T}}(t-\tau), \dot{x}^{\mathrm{T}}(t-\tau), \int_{t-\tau}^{t} x^{\mathrm{T}}(s) d s\right. \\
& \left.\int_{t-\tau}^{t} \int_{s}^{t} x^{\mathrm{T}}(u) d u d s\right]
\end{aligned}
$$

and $e_{i} \in R^{10 n \times n}(i=1,2, \ldots, 10)$ means the block entry matrices; for example, $e_{3}^{\mathrm{T}} \zeta(t)=x(t-h), e_{9}^{\mathrm{T}} \zeta(t)=\int_{t-\tau}^{t} x(s) d s$.

Using Lemmas 1 and 2 to four integral terms $\xi_{1}(t), \xi_{2}(t)$, $\xi_{3}(t)$, and $\xi_{4}(t)$ in (19), we have

$$
\begin{aligned}
-h \xi_{1}(t) & \leq-\phi_{1,1}^{\mathrm{T}}(t) D \phi_{1,1}(t)-3 \phi_{1,2}^{\mathrm{T}}(t) D \phi_{1,2}(t), \\
-\frac{h^{2}}{2} \xi_{2}(t) & \leq-\phi_{2,1}^{\mathrm{T}}(t) T \phi_{2,1}(t)-2 \phi_{2,2}^{\mathrm{T}}(t) T \phi_{2,2}(t), \\
-\tau \xi_{3}(t) & \leq-\phi_{3,1}^{\mathrm{T}}(t) \bar{D} \phi_{3,1}(t)-3 \phi_{3,2}^{\mathrm{T}}(t) \bar{D} \phi_{3,2}(t), \\
-\frac{\tau^{2}}{2} \xi_{4}(t) & \leq-\phi_{4,1}^{\mathrm{T}}(t) \bar{T} \phi_{4,1}(t)-2 \phi_{4,2}^{\mathrm{T}}(t) \bar{T} \phi_{4,2}(t),
\end{aligned}
$$

where

$$
\begin{aligned}
& \varphi_{1,1}(t)=\int_{t-h}^{t} v_{2}(s) d s=\left[\begin{array}{c}
\int_{t-h}^{t} x(s) d s \\
x(t)-x(t-h)
\end{array}\right] \\
& =\left[\begin{array}{ll}
e_{5} & e_{1}-e_{3}
\end{array}\right]^{\mathrm{T}} \zeta(t), \\
& \varphi_{1,2}(t)=\frac{2}{h} \int_{t-h}^{t} \int_{s}^{t} v_{2}(u) d u d s-\int_{t-h}^{t} v_{2}(s) d s \\
& =\left[\begin{array}{c}
\frac{2}{h} \int_{t-h}^{t} \int_{s}^{t} x(u) d u d s-\int_{t-h}^{t} x(s) d s \\
x(t)+x(t-h)-\frac{2}{h} \int_{t-h}^{t} x(s) d s
\end{array}\right] \\
& =\left[\frac{2}{h} e_{6}-e_{5} e_{1}+e_{3}-\frac{2}{h} e_{5}\right]^{\mathrm{T}} \zeta(t) \text {, } \\
& \varphi_{2,1}(t)=\int_{t-h}^{t} \int_{s}^{t} \dot{x}(u) d u d s=h x(t)-\int_{t-h}^{t} x(s) d s \\
& =\left(h e_{1}-e_{5}\right)^{\mathrm{T}} \zeta(t) \text {, } \\
& \varphi_{2,2}(t)=\frac{3}{h} \int_{t-h}^{t} \int_{s}^{t} \int_{u}^{t} \dot{x}(v) d v d u d s \\
& -\int_{t-h}^{t} \int_{s}^{t} \dot{x}(u) d u d s \\
& =\frac{h}{2} x(t)+\int_{t-h}^{t} x(s) d s
\end{aligned}
$$

$$
\begin{aligned}
& -\frac{3}{h} \int_{t-h}^{t} \int_{s}^{t} x(u) d u d s \\
& =\left(\frac{h}{2} e_{1}+e_{5}-\frac{3}{h} e_{6}\right)^{\mathrm{T}} \zeta(t) \text {, } \\
& \varphi_{3,1}(t)=\int_{t-\tau}^{t} v_{2}(s) d s=\left[\begin{array}{c}
\int_{t-\tau}^{t} x(s) d s \\
x(t)-x(t-\tau)
\end{array}\right] \\
& =\left[\begin{array}{ll}
e_{9} & e_{1}-e_{7}
\end{array}\right]^{\mathrm{T}} \zeta(t), \\
& \varphi_{3,2}(t)=\frac{2}{\tau} \int_{t-\tau}^{t} \int_{s}^{t} v_{2}(u) d u d s-\int_{t-\tau}^{t} v_{2}(s) d s \\
& =\left[\begin{array}{c}
\frac{2}{\tau} \int_{t-\tau}^{t} \int_{s}^{t} x(u) d u d s-\int_{t-\tau}^{t} x(s) d s \\
x(t)+x(t-\tau)-\frac{2}{\tau} \int_{t-\tau}^{t} x(s) d s
\end{array}\right] \\
& =\left[\frac{2}{\tau} e_{10}-e_{9} e_{1}+e_{7}-\frac{2}{\tau} e_{9}\right]^{\mathrm{T}} \zeta(t) \text {, } \\
& \varphi_{4,1}(t)=\int_{t-\tau}^{t} \int_{s}^{t} \dot{x}(u) d u d s=\tau x(t)-\int_{t-\tau}^{t} x(s) d s \\
& =\left(\tau e_{1}-e_{9}\right)^{\mathrm{T}} \zeta(t), \\
& \varphi_{4,2}(t)=\frac{3}{\tau} \int_{t-\tau}^{t} \int_{s}^{t} \int_{u}^{t} \dot{x}(v) d v d u d s \\
& -\int_{t-\tau}^{t} \int_{s}^{t} \dot{x}(u) d u d s \\
& =\frac{\tau}{2} x(t)+\int_{t-\tau}^{t} x(s) d s \\
& -\frac{3}{\tau} \int_{t-\tau}^{t} \int_{s}^{t} x(u) d u d s \\
& =\left(\frac{\tau}{2} e_{1}+e_{9}-\frac{3}{\tau} e_{10}\right)^{\mathrm{T}} \zeta(t) .
\end{aligned}
$$

Combining (21)-(24), (19) can be rewritten as

$$
\begin{aligned}
\dot{V} \leq & \zeta^{\mathrm{T}}(t)\left(\Xi_{0}+\Xi_{1}+\Xi_{2,1}+\Xi_{2,2}+\Xi_{3}+\Xi_{4,1}+\Xi_{4,2}\right) \\
& \cdot \zeta(t)=\zeta^{\mathrm{T}}(t) \Xi \zeta(t),
\end{aligned}
$$


6

Mathematical Problems in Engineering

where

$$
\begin{aligned}
& \Xi_{1}=-\left[\begin{array}{ll}
e_{5} & e_{1}-e_{3}
\end{array}\right] D\left[\begin{array}{ll}
e_{5} & e_{1}-e_{3}
\end{array}\right]^{\mathrm{T}}-3\left[\left(\frac{2}{h}\right) e_{6}-e_{5} e_{1}+e_{3}-\left(\frac{2}{h}\right) e_{5}\right] D\left[\left(\frac{2}{h}\right) e_{6}-e_{5} e_{1}+e_{3}-\left(\frac{2}{h}\right) e_{5}\right]^{\mathrm{T}}, \\
& \Xi_{2,1}=-\left[h e_{1}-e_{5}\right] T\left[h e_{1}-e_{5}\right]^{\mathrm{T}}, \\
& \Xi_{2,2}=-2\left[\left(\frac{h}{2}\right) e_{1}+e_{5}-\left(\frac{3}{h}\right) e_{6}\right] T\left[\left(\frac{h}{2}\right) e_{1}+e_{5}-\left(\frac{3}{h}\right) e_{6}\right]^{\mathrm{T}} \text {, } \\
& \Xi_{3}=-\left[\begin{array}{ll}
e_{9} & e_{1}-e_{7}
\end{array}\right] \bar{D}\left[\begin{array}{ll}
e_{9} & e_{1}-e_{7}
\end{array}\right]^{\mathrm{T}}-3\left[\left(\frac{2}{\tau}\right) e_{10}-e_{9} e_{1}+e_{7}-\left(\frac{2}{\tau}\right) e_{9}\right] \bar{D}\left[\left(\frac{2}{\tau}\right) e_{10}-e_{9} e_{1}+e_{7}-\left(\frac{2}{\tau}\right) e_{9}\right]^{\mathrm{T}} \text {, } \\
& \Xi_{4,1}=-\left[\tau e_{1}-e_{9}\right] \bar{T}\left[\tau e_{1}-e_{9}\right]^{\mathrm{T}} \\
& \Xi_{4,2}=-2\left[\left(\frac{\tau}{2}\right) e_{1}+e_{9}-\left(\frac{3}{\tau}\right) e_{10}\right] \bar{T}\left[\left(\frac{\tau}{2}\right) e_{1}+e_{9}-\left(\frac{3}{\tau}\right) e_{10}\right]^{\mathrm{T}} \\
& \Xi=\Xi_{0}+\Xi_{1}+\Xi_{2,1}+\Xi_{2,2}+\Xi_{3}+\Xi_{4,1}+\Xi_{4,2} \text {. }
\end{aligned}
$$

Then, a new stability condition for system (9) can be described as

$$
\zeta^{\mathrm{T}}(t) \Xi \zeta(t)<0 \quad \text { st. } \Gamma(\lambda) \zeta(t)=0, \zeta(t) \neq 0,
$$

where $\Gamma(\lambda)=\left[\begin{array}{llll}A(\lambda) & -I & A_{d}(\lambda) & B(\lambda) K(\lambda)\end{array}\right]\left[\begin{array}{llll}e_{1} & e_{2} & e_{3} & e_{7}\end{array}\right]^{\mathrm{T}}$.

Based on Lemma 3, formula (28) can be rewritten as

$$
\Xi+L \Gamma(\lambda)+\Gamma(\lambda)^{\mathrm{T}} L^{\mathrm{T}}<0,
$$

where $L=a_{1} e_{1} L_{0}+e_{2} L_{0}+a_{2} e_{3} L_{0}$.
Now, let $L_{0}=X^{-\mathrm{T}}$ and $K(\lambda)=M(\lambda) X^{-1}$. Applying matrix inequality (29) to the left multiplication $\operatorname{diag}[X, X, X, X, X, X, X, X, X, X]^{\mathrm{T}}$ and right multiplication $\operatorname{diag}[X, X, X, X, X, X, X, X, X, X]$, we have

$$
\widehat{\Xi}+\widehat{L} \widehat{\Gamma}(\lambda)+\widehat{\Gamma}(\lambda)^{\mathrm{T}} \widehat{L}^{\mathrm{T}}<0
$$

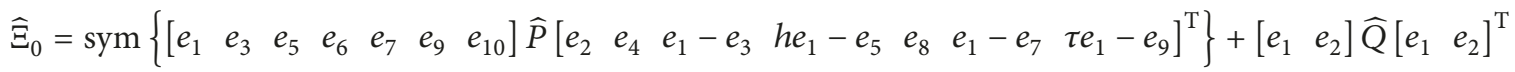

$$
\begin{aligned}
& -\left[\begin{array}{ll}
e_{3} & e_{4}
\end{array}\right] \widehat{Q}\left[\begin{array}{ll}
e_{3} & e_{4}
\end{array}\right]^{\mathrm{T}}+h^{2}\left[\begin{array}{ll}
e_{1} & e_{2}
\end{array}\right] \widehat{D}\left[\begin{array}{ll}
e_{1} & e_{2}
\end{array}\right]^{\mathrm{T}}+\left(\frac{h^{2}}{2}\right)^{2} e_{2} \widehat{T} e_{2}^{\mathrm{T}}+\left[\begin{array}{ll}
e_{1} & e_{2}
\end{array}\right] \widehat{\bar{Q}}\left[\begin{array}{ll}
e_{1} & e_{2}
\end{array}\right]^{\mathrm{T}}-\left[\begin{array}{ll}
e_{7} & e_{8}
\end{array}\right] \widehat{\bar{Q}}\left[\begin{array}{ll}
e_{7} & e_{8}
\end{array}\right]^{\mathrm{T}} \\
& +\tau^{2}\left[\begin{array}{ll}
e_{1} & e_{2}
\end{array}\right] \widehat{\bar{D}}\left[e_{1} e_{2}\right]^{\mathrm{T}}+\left(\frac{\tau^{2}}{2}\right)^{2} e_{2} \widehat{\bar{T}} e_{2}{ }^{\mathrm{T}} \\
& \widehat{\Xi}_{1}=-\left[\begin{array}{ll}
e_{5} & e_{1}-e_{3}
\end{array}\right] \widehat{D}\left[\begin{array}{ll}
e_{5} & e_{1}-e_{3}
\end{array}\right]^{\mathrm{T}}-3\left[\left(\frac{2}{h}\right) e_{6}-e_{5} e_{1}+e_{3}-\left(\frac{2}{h}\right) e_{5}\right] \widehat{D}\left[\left(\frac{2}{h}\right) e_{6}-e_{5} e_{1}+e_{3}-\left(\frac{2}{h}\right) e_{5}\right]^{\mathrm{T}} \text {, } \\
& \widehat{\Xi}_{2,1}=-\left[h e_{1}-e_{5}\right] \widehat{T}\left[h e_{1}-e_{5}\right]^{\mathrm{T}}, \\
& \widehat{\Xi}_{2,2}=-2\left[\left(\frac{h}{2}\right) e_{1}+e_{5}-\left(\frac{3}{h}\right) e_{6}\right] \widehat{T}\left[\left(\frac{h}{2}\right) e_{1}+e_{5}-\left(\frac{3}{h}\right) e_{6}\right]^{\mathrm{T}}, \\
& \widehat{\Xi}_{3}=-\left[\begin{array}{ll}
e_{9} & e_{1}-e_{7}
\end{array}\right] \widehat{\bar{D}}\left[\begin{array}{ll}
e_{9} & e_{1}-e_{7}
\end{array}\right]^{\mathrm{T}}-3\left[\left(\frac{2}{\tau}\right) e_{10}-e_{9} e_{1}+e_{7}-\left(\frac{2}{\tau}\right) e_{9}\right] \widehat{\bar{D}}\left[\left(\frac{2}{\tau}\right) e_{10}-e_{9} e_{1}+e_{7}-\left(\frac{2}{\tau}\right) e_{9}\right]^{\mathrm{T}} \text {, } \\
& \widehat{\Xi}_{4,1}=-\left[\tau e_{1}-e_{9}\right] \widehat{\bar{T}}\left[\tau e_{1}-e_{9}\right]^{\mathrm{T}}, \\
& \widehat{\Xi}_{4,2}=-2\left[\left(\frac{\tau}{2}\right) e_{1}+e_{9}-\left(\frac{3}{\tau}\right) e_{10}\right] \widehat{\bar{T}}\left[\left(\frac{\tau}{2}\right) e_{1}+e_{9}-\left(\frac{3}{\tau}\right) e_{10}\right]^{\mathrm{T}} \text {, } \\
& \widehat{\Xi}=\widehat{\Xi}_{0}+\widehat{\Xi}_{1}+\widehat{\Xi}_{2,1}+\widehat{\Xi}_{2,2}+\widehat{\Xi}_{3}+\widehat{\Xi}_{4,1}+\widehat{\Xi}_{4,2} \text {, }
\end{aligned}
$$




$$
\begin{aligned}
& \widehat{P}=\left(\operatorname{diag}[X, X, X, X, X, X, X]^{\mathrm{T}}\right) P(\operatorname{diag}[X, X, X, X, X, X, X]), \\
& \widehat{Q}=\left(\operatorname{diag}[X, X]^{\mathrm{T}}\right) Q(\operatorname{diag}[X, X]), \\
& \widehat{\bar{Q}}=\left(\operatorname{diag}[X, X]^{\mathrm{T}}\right) \bar{Q}(\operatorname{diag}[X, X]), \\
& \widehat{D}=\left(\operatorname{diag}[X, X]^{\mathrm{T}}\right) D(\operatorname{diag}[X, X]), \\
& \widehat{\bar{D}}=\left(\operatorname{diag}[X, X]^{\mathrm{T}}\right) \widehat{D}(\operatorname{diag}[X, X]) \\
& \widehat{T}=X^{\mathrm{T}} T X \\
& \widehat{\bar{T}}=X^{\mathrm{T}} \bar{T} X .
\end{aligned}
$$

Since

$$
\begin{aligned}
& \left(\operatorname{diag}[X, X, X, X, X, X, X, X, X, X]^{\mathrm{T}}\right) L \Gamma(\lambda) \\
& \cdot(\operatorname{diag}[X, X, X, X, X, X, X, X, X, X])=\left(a_{1} e_{1}+e_{2}\right. \\
& \left.+a_{2} e_{3}\right)\left[A(\lambda) X-X \quad A_{d}(\lambda) X \quad B(\lambda) M(\lambda)\right]\left[e_{1},\right. \\
& \left.e_{2}, e_{3}, e_{7}\right]^{\mathrm{T}}=\widehat{L} \widehat{\Gamma}(\lambda),
\end{aligned}
$$

we have $\widehat{L}=a_{1} e_{1}+e_{2}+a_{2} e_{3}$ and $\widehat{\Gamma}(\lambda)=$ $\left[A(\lambda) X-X \quad A_{d}(\lambda) X \quad B(\lambda) M(\lambda)\right]\left[e_{1}, e_{2}, e_{3}, e_{7}\right]^{\mathrm{T}}$.

Thus, we transform inequality (29) into the form of linear matrix inequality, which is defined in (14). The whole proof is completed.
Theorem 4 is dependent on time-varying information $\lambda$, which cannot be computed by LMI. In the following analysis, our goal is to convert Theorem 4 into LMI.

Theorem 5. Consider the closed-loop system (9) and given scalars $h$ and $\tau$ to meet $h>0, \tau>0$, the system is asymptotically stable, if there exist scalars $a_{1}$ and $a_{2}$, matrices $M_{j}, X$, and positive definite symmetric matrices $\widehat{P}, \widehat{Q}, \widehat{D}, \widehat{T}, \widehat{\bar{Q}}$, $\widehat{\bar{D}}$, and $\widehat{\bar{T}}$, such that the following LMI holds:

$$
\begin{gathered}
\widehat{\Xi}+\widehat{L} \widehat{\Gamma}(i, i)+\widehat{\Gamma}(i, i)^{\mathrm{T}} \widehat{L}^{\mathrm{T}}<0, \quad i=1 \cdots r, \\
\widehat{\Xi}+\widehat{L} \widehat{\Gamma}(i, j)+\widehat{\Gamma}(i, j)^{\mathrm{T}} \widehat{L}^{\mathrm{T}}+\widehat{\Xi}+\widehat{L} \widehat{\Gamma}(j, i) \\
+\widehat{\Gamma}(j, i)^{\mathrm{T}} \widehat{L}^{\mathrm{T}}<0 \quad 1 \leq i<j \leq r,
\end{gathered}
$$

where

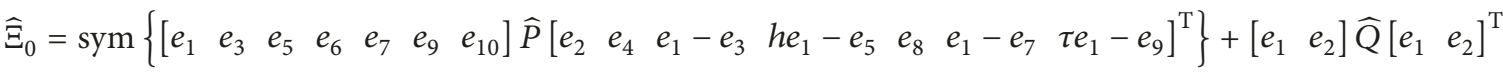

$$
\begin{aligned}
& -\left[\begin{array}{ll}
e_{3} & e_{4}
\end{array}\right] \widehat{\mathrm{Q}}\left[\begin{array}{ll}
e_{3} & e_{4}
\end{array}\right]^{\mathrm{T}}+h^{2}\left[\begin{array}{ll}
e_{1} & e_{2}
\end{array}\right] \widehat{D}\left[\begin{array}{ll}
e_{1} & e_{2}
\end{array}\right]^{\mathrm{T}}+\left(\frac{h^{2}}{2}\right)^{2} e_{2} \widehat{T} e_{2}{ }^{\mathrm{T}}+\left[\begin{array}{ll}
e_{1} & e_{2}
\end{array}\right] \widehat{\bar{Q}}\left[\begin{array}{ll}
e_{1} & e_{2}
\end{array}\right]^{\mathrm{T}}-\left[\begin{array}{ll}
e_{7} & e_{8}
\end{array}\right] \widehat{\bar{Q}}\left[\begin{array}{ll}
e_{7} & e_{8}
\end{array}\right]^{\mathrm{T}} \\
& +\tau^{2}\left[\begin{array}{ll}
e_{1} & e_{2}
\end{array}\right] \widehat{\bar{D}}\left[\begin{array}{ll}
e_{1} & e_{2}
\end{array}\right]^{\mathrm{T}}+\left(\frac{\tau^{2}}{2}\right)^{2} e_{2} \widehat{\bar{T}} e_{2}^{\mathrm{T}} \\
& \widehat{\Xi}_{1}=-\left[\begin{array}{ll}
e_{5} & e_{1}-e_{3}
\end{array}\right] \widehat{D}\left[\begin{array}{ll}
e_{5} & e_{1}-e_{3}
\end{array}\right]^{\mathrm{T}}-3\left[\left(\frac{2}{h}\right) e_{6}-e_{5} e_{1}+e_{3}-\left(\frac{2}{h}\right) e_{5}\right] \widehat{D}\left[\left(\frac{2}{h}\right) e_{6}-e_{5} e_{1}+e_{3}-\left(\frac{2}{h}\right) e_{5}\right]^{\mathrm{T}} \text {, } \\
& \widehat{\Xi}_{2,1}=-\left[h e_{1}-e_{5}\right] \widehat{T}\left[h e_{1}-e_{5}\right]^{\mathrm{T}} \text {, } \\
& \widehat{\Xi}_{2,2}=-2\left[\left(\frac{h}{2}\right) e_{1}+e_{5}-\left(\frac{3}{h}\right) e_{6}\right] \widehat{T}\left[\left(\frac{h}{2}\right) e_{1}+e_{5}-\left(\frac{3}{h}\right) e_{6}\right]^{\mathrm{T}}, \\
& \widehat{\Xi}_{3}=-\left[\begin{array}{ll}
e_{9} & e_{1}-e_{7}
\end{array}\right] \widehat{\bar{D}}\left[\begin{array}{ll}
e_{9} & e_{1}-e_{7}
\end{array}\right]^{\mathrm{T}}-3\left[\left(\frac{2}{\tau}\right) e_{10}-e_{9} e_{1}+e_{7}-\left(\frac{2}{\tau}\right) e_{9}\right] \widehat{\bar{D}}\left[\left(\frac{2}{\tau}\right) e_{10}-e_{9} e_{1}+e_{7}-\left(\frac{2}{\tau}\right) e_{9}\right]^{\mathrm{T}} \text {, } \\
& \widehat{\Xi}_{4,1}=-\left[\tau e_{1}-e_{9}\right] \hat{\bar{T}}\left[\tau e_{1}-e_{9}\right]^{\mathrm{T}} \text {, } \\
& \widehat{\Xi}_{4,2}=-2\left[\left(\frac{\tau}{2}\right) e_{1}+e_{9}-\left(\frac{3}{\tau}\right) e_{10}\right] \widehat{\bar{T}}\left[\left(\frac{\tau}{2}\right) e_{1}+e_{9}-\left(\frac{3}{\tau}\right) e_{10}\right]^{\mathrm{T}} \text {, }
\end{aligned}
$$




$$
\begin{aligned}
& \widehat{\Xi}=\widehat{\Xi}_{0}+\widehat{\Xi}_{1}+\widehat{\Xi}_{2,1}+\widehat{\Xi}_{2,2}+\widehat{\Xi}_{3}+\widehat{\Xi}_{4,1}+\widehat{\Xi}_{4,2}, \\
& \widehat{L}=a_{1} e_{1}+e_{2}+a_{2} e_{3} \text {, } \\
& \widehat{\Gamma}(i, j)=\left[\begin{array}{llll}
A_{i} X & -X & A_{d i} X & B_{i} M_{j}
\end{array}\right]\left[e_{1}, e_{2}, e_{3}, e_{7}\right]^{\mathrm{T}} .
\end{aligned}
$$

In addition, the gain matrix of state feedback controller can be described as

$$
K_{j}=M_{j} X^{-1}
$$

Proof. Consider the following forms and substitute them into inequality (14):

$$
\begin{gathered}
A(\lambda)=\sum_{i=1}^{r} \lambda_{i}(\theta(t)) A_{i} \\
A_{d}(\lambda)=\sum_{i=1}^{r} \lambda_{i}(\theta(t)) A_{d i} \\
B(\lambda)=\sum_{i=1}^{r} \lambda_{i}(\theta(t)) B_{i} \\
M(\lambda)=\sum_{i=1}^{r} \lambda_{i}(\theta(t)) M_{i}
\end{gathered}
$$

Thus, inequality (14) can be rewritten as

$$
\begin{aligned}
& \sum_{i=1}^{r} \sum_{j=1}^{r} \lambda_{i}(\theta(t)) \lambda_{j}(\theta(t))\left(\widehat{\Xi}+\widehat{L} \widehat{\Gamma}(i, j)+\widehat{\Gamma}(i, j)^{\mathrm{T}} \widehat{L}^{\mathrm{T}}\right) \\
& \quad<0
\end{aligned}
$$

where $\widehat{\Gamma}(i, j)=\left[\begin{array}{llll}A_{i} X & -X & A_{d i} X & B_{i} M_{j}\end{array}\right]\left[e_{1}, e_{2}, e_{3}, e_{7}\right]^{\mathrm{T}}$.

Now we can rewrite (37) as

$$
\begin{aligned}
& \sum_{i=1}^{r} \lambda_{i}^{2}(\theta(t))\left(\widehat{\Xi}+\widehat{L} \widehat{\Gamma}(i, i)+\widehat{\Gamma}(i, i)^{\mathrm{T}} \widehat{L}^{\mathrm{T}}\right) \\
& \quad+\sum_{i=1}^{r} \sum_{i<j}^{r} \lambda_{i}(\theta(t)) \lambda_{j}(\theta(t)) \times(\widehat{\Xi}+\widehat{L} \widehat{\Gamma}(i, j) \\
& \left.\quad+\widehat{\Gamma}(i, j)^{\mathrm{T}} \widehat{L}^{\mathrm{T}}+\widehat{\Xi}+\widehat{L} \widehat{\Gamma}(j, i)+\widehat{\Gamma}(j, i)^{\mathrm{T}} \widehat{L}^{\mathrm{T}}\right)<0 .
\end{aligned}
$$

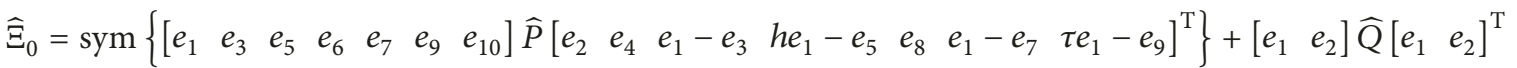

$$
\begin{aligned}
& -\left[\begin{array}{ll}
e_{3} & e_{4}
\end{array}\right] \widehat{\mathrm{Q}}\left[\begin{array}{ll}
e_{3} & e_{4}
\end{array}\right]^{\mathrm{T}}+h^{2}\left[\begin{array}{ll}
e_{1} & e_{2}
\end{array}\right] \widehat{D}\left[\begin{array}{ll}
e_{1} & e_{2}
\end{array}\right]^{\mathrm{T}}+\left[\begin{array}{ll}
e_{1} & e_{2}
\end{array}\right] \widehat{\bar{Q}}\left[\begin{array}{ll}
e_{1} & e_{2}
\end{array}\right]^{\mathrm{T}}-\left[\begin{array}{ll}
e_{7} & e_{8}
\end{array}\right] \widehat{\bar{Q}}\left[\begin{array}{ll}
e_{7} & e_{8}
\end{array}\right]^{\mathrm{T}}+\tau^{2}\left[\begin{array}{ll}
e_{1} & e_{2}
\end{array}\right] \widehat{\bar{D}}\left[\begin{array}{ll}
e_{1} & e_{2}
\end{array}\right]^{\mathrm{T}}, \\
& \widehat{\Xi}_{1}=-\left[\begin{array}{ll}
e_{5} & e_{1}-e_{3}
\end{array}\right] \widehat{D}\left[\begin{array}{ll}
e_{5} & e_{1}-e_{3}
\end{array}\right]^{\mathrm{T}}-3\left[\left(\frac{2}{h}\right) e_{6}-e_{5} e_{1}+e_{3}-\left(\frac{2}{h}\right) e_{5}\right] \widehat{D}\left[\left(\frac{2}{h}\right) e_{6}-e_{5} e_{1}+e_{3}-\left(\frac{2}{h}\right) e_{5}\right]^{\mathrm{T}} \text {, } \\
& \widehat{\Xi}_{3}=-\left[\begin{array}{ll}
e_{9} & e_{1}-e_{7}
\end{array}\right] \widehat{\bar{D}}\left[\begin{array}{ll}
e_{9} & e_{1}-e_{7}
\end{array}\right]^{\mathrm{T}}-3\left[\left(\frac{2}{\tau}\right) e_{10}-e_{9} e_{1}+e_{7}-\left(\frac{2}{\tau}\right) e_{9}\right] \widehat{\bar{D}}\left[\left(\frac{2}{\tau}\right) e_{10}-e_{9} e_{1}+e_{7}-\left(\frac{2}{\tau}\right) e_{9}\right]^{\mathrm{T}} \text {, } \\
& \widehat{\Xi}=\widehat{\Xi}_{0}+\widehat{\Xi}_{1}+\widehat{\Xi}_{3} \text {, }
\end{aligned}
$$

where

$$
\begin{aligned}
& \widehat{\Xi}+\widehat{L} \widehat{\Gamma}(i, i)+\widehat{\Gamma}(i, i)^{\mathrm{T}} \widehat{L}^{\mathrm{T}}<0, \quad i=1 \cdots r, \\
& \widehat{\Xi}+\widehat{L} \widehat{\Gamma}(i, j)+\widehat{\Gamma}(i, j)^{\mathrm{T}} \widehat{L}^{\mathrm{T}}+\widehat{\Xi}+\widehat{L} \widehat{\Gamma}(j, i) \\
& +\widehat{\Gamma}(j, i)^{\mathrm{T}} \widehat{L}^{\mathrm{T}}<0 \quad 1 \leq i<j \leq r .
\end{aligned}
$$

Remark 6. T-S fuzzy time-delay systems have been widely studied in previous papers. However, the existing literature only considers one delay factor, for example, state delay or input delay. Currently, a few results are presented for T-S fuzzy system with state and input delays. Unlike the existing results, a new Lyapunov-Krasovskii functional including integral terms is employed to derive less conservative bility conditions. In the next section, some simulation proposed method.

In order to show the effectiveness of the Lyapunov-Krasovskii functional with triple integral terms and Wirtingerbased double integral inequality, we use the LyapunovKrasovskii functional of (17) without triple integral terms to derive new stabilization conditions. Now we give the

Corollary 7. Consider the closed-loop system (9) and given scalars $h$ and $\tau$ to meet $h>0, \tau>0$, the system is asymptotically stable, if there exist scalars $a_{1}$ and $a_{2}$, matrices $M_{j}, X$, and positive definite symmetric matrices $\widehat{P}, \widehat{Q}, \widehat{D}, \widehat{\bar{Q}}, \widehat{\bar{D}}$, such that the following LMI holds:

$$
\begin{gathered}
\widehat{\Xi}+\widehat{L} \widehat{\Gamma}(i, i)+\widehat{\Gamma}(i, i)^{\mathrm{T}} \widehat{L}^{\mathrm{T}}<0, \quad i=1 \cdots r, \\
\widehat{\Xi}+\widehat{L} \widehat{\Gamma}(i, j)+\widehat{\Gamma}(i, j)^{\mathrm{T}} \widehat{L}^{\mathrm{T}}+\widehat{\Xi}+\widehat{L} \widehat{\Gamma}(j, i) \\
+\widehat{\Gamma}(j, i)^{\mathrm{T}} \widehat{L}^{\mathrm{T}}<0 \quad 1 \leq i<j \leq r,
\end{gathered}
$$

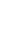




$$
\begin{aligned}
& \widehat{L}=a_{1} e_{1}+e_{2}+a_{2} e_{3}, \\
& \widehat{\Gamma}(i, j)=\left[\begin{array}{llll}
A_{i} X & -X & A_{d i} X & B_{i} M_{j}
\end{array}\right]\left[e_{1}, e_{2}, e_{3}, e_{7}\right]^{\mathrm{T}} \text {. }
\end{aligned}
$$

In addition, the gain matrix of state feedback controller can be described as

$$
K_{j}=M_{j} X^{-1} .
$$

\section{Numerical Examples}

In this section, we provide three numerical examples to illustrate the effectiveness of the stabilization criteria developed by this paper. The first examples show the improvement of our results. The second and third examples are used to demonstrate the effectiveness of the controller design.

Example 1. Consider a two-rule T-S fuzzy system (2). The system matrices are given as follows:

$$
\begin{aligned}
A_{1} & =\left[\begin{array}{cc}
0 & 1 \\
1 & -2
\end{array}\right], \\
A_{2} & =\left[\begin{array}{cc}
1 & 0 \\
1 & -2
\end{array}\right], \\
B_{1} & =B_{2}=\left[\begin{array}{l}
1 \\
0
\end{array}\right], \\
A_{d 1} & =\left[\begin{array}{ll}
0 & 0 \\
0 & 0
\end{array}\right], \\
A_{d 2} & =\left[\begin{array}{ll}
0 & 0 \\
0 & 0
\end{array}\right] .
\end{aligned}
$$

In this example, only input delay is considered. When the parameters are defined as $a_{1}=a_{2}=0.1$, the maximum input delay and the corresponding gain matrix of the system are obtained by Theorem 5. Meanwhile, the proposed method is compared with that in [42], as shown in Table 1. Clearly, the method of Theorem 5 is more superior than the existing results. Moreover, we cannot find feasible solution by Corollary 7. Therefore, the Lyapunov-Krasovskii functional with triple integral terms and Wirtinger-based double integral inequality are important to reduce the conservativeness of stability analysis for T-S fuzzy systems with state and input delays.

Example 2. Consider the following complex nonlinear continuous-time system studied in [43]:

$$
\begin{aligned}
\dot{x}_{1}(t)= & x_{1}(t)+x_{2}(t)+\sin x_{3}(t)-0.1 x_{4}(t) \\
& +\left(x_{1}^{2}(t)+1\right) u(t), \\
\dot{x}_{2}(t)= & x_{1}(t)-2 x_{2}(t),
\end{aligned}
$$

$$
\begin{aligned}
& \dot{x}_{3}(t)=x_{1}(t)+x_{1}^{2}(t) x_{2}(t)-0.3 x_{3}(t), \\
& \dot{x}_{4}(t)=\sin x_{3}(t)-x_{4}(t), \\
& y_{1}(t)=x_{2}(t)+\left(x_{1}^{2}(t)+1\right) x_{4}(t), \\
& y_{2}(t)=x_{1}(t)
\end{aligned}
$$

where $a$ and $b$ are positive numbers, and assume $x_{1}(t) \in$ $[-a, a], x_{3}(t) \in[-b, b]$.

The nonlinear system is exactly represented by the following T-S fuzzy model.

Plant Rule 1. If $x_{1}(t)$ is $M_{1}^{1}$ and $x_{3}(t)$ is $M_{3}^{1}$, then

$$
\begin{aligned}
& \dot{x}(t)=A_{1} x(t)+B_{1} u(t) \\
& y(t)=C_{1} x(t) .
\end{aligned}
$$

Plant Rule 2. If $x_{1}(t)$ is $M_{1}^{1}$ and $x_{3}(t)$ is $M_{3}^{2}$, then

$$
\begin{aligned}
& \dot{x}(t)=A_{2} x(t)+B_{2} u(t) \\
& y(t)=C_{2} x(t) .
\end{aligned}
$$

Plant Rule 3. If $x_{1}(t)$ is $M_{1}^{2}$ and $x_{3}(t)$ is $M_{3}^{1}$, then

$$
\begin{aligned}
& \dot{x}(t)=A_{3} x(t)+B_{3} u(t) \\
& y(t)=C_{3} x(t) .
\end{aligned}
$$

Plant Rule 4. If $x_{1}(t)$ is $M_{1}^{2}$ and $x_{3}(t)$ is $M_{3}^{2}$, then

$$
\begin{aligned}
& \dot{x}(t)=A_{4} x(t)+B_{4} u(t) \\
& y(t)=C_{4} x(t),
\end{aligned}
$$

where

$$
A_{1}=\left[\begin{array}{cccc}
1 & 1 & 1 & -0.1 \\
1 & -2 & 0 & 0 \\
1 & a^{2} & -0.3 & 0 \\
0 & 0 & 1 & -1
\end{array}\right],
$$


TABLE 1: Maximum allowable time delay and feedback gains.

\begin{tabular}{lcc}
\hline Literature & $\tau$ & Feedback gains \\
\hline$[42]$ & 0.5953 & $K_{1}=\left(\begin{array}{ll}-0.9379 & -0.6779\end{array}\right)$ \\
& & $K_{2}=\left(\begin{array}{ll}-0.8903 & -0.2171\end{array}\right)$ \\
Theorem 5 & 0.9799 & $K_{1}=\left(\begin{array}{ll}-0.0030 & -1.0151\end{array}\right)$ \\
& & $K_{2}=\left(\begin{array}{ll}-1.0094 & 0.0171\end{array}\right)$
\end{tabular}

$A_{2}=\left[\begin{array}{cccc}1 & 1 & \frac{(\sin b)}{b} & -0.1 \\ 1 & -2 & 0 & 0 \\ 1 & a^{2} & -0.3 & 0 \\ 0 & 0 & \frac{(\sin b)}{b} & -1\end{array}\right]$

$A_{3}=\left[\begin{array}{cccc}1 & 1 & 1 & -0.1 \\ 1 & -2 & 0 & 0 \\ 1 & 0 & -0.3 & 0 \\ 0 & 0 & 1 & -1\end{array}\right]$,

$A_{4}=\left[\begin{array}{cccc}1 & 1 & \frac{(\sin b)}{b} & -0.1 \\ 1 & -2 & 0 & 0 \\ 1 & 0 & -0.3 & 0 \\ 0 & 0 & \frac{(\sin b)}{b} & -1\end{array}\right]$

$B_{1}=B_{2}=\left[\begin{array}{c}1+a^{2} \\ 0 \\ 0 \\ 0\end{array}\right]$,

$B_{3}=B_{4}=\left[\begin{array}{l}1 \\ 0 \\ 0 \\ 0\end{array}\right]$.

To verify the effectiveness of the proposed method, the other system matrices are given as

$$
\begin{aligned}
& A_{d 1}=A_{d 2}=\left[\begin{array}{llll}
0.1 & 0 & 0 & 0 \\
0 & 0 & 0 & 0 \\
0 & 0 & 0 & 0 \\
0 & 0 & 0 & 0
\end{array}\right], \\
& A_{d 3}=A_{d 4}=\left[\begin{array}{llll}
0.1 & 0 & 0 & 0 \\
0 & 0 & 0 & 0 \\
0 & 0 & 0 & 0 \\
0 & 0 & 0 & 0
\end{array}\right] .
\end{aligned}
$$

In particular, assuming $a=1.4$ and $b=0.7$ and setting parameters $a_{1}=1$ and $a_{2}=0.01$, we can get the maximum allowable delays of input and state as $h_{\max }=68$ and $\tau_{\max }=$ 0.277 , respectively. However, we cannot find feasible solution by Corollary 7. According to Theorem 5, taking $h=10$ and $\tau=0.27$, we have the following feedback gains:

$$
\begin{aligned}
& K_{1}=\left[\begin{array}{llll}
-0.7593 & -0.3889 & -0.3436 & 0.0275
\end{array}\right], \\
& K_{2}=\left[\begin{array}{llll}
-0.7391 & -0.3767 & -0.3174 & 0.0265
\end{array}\right], \\
& K_{3}=\left[\begin{array}{llll}
-1.9484 & -0.5514 & -0.8764 & 0.0701
\end{array}\right], \\
& K_{4}=\left[\begin{array}{llll}
-1.9354 & -0.5713 & -0.8440 & 0.0692
\end{array}\right]
\end{aligned}
$$

For simulation, we choose

$$
\begin{aligned}
& \lambda_{1}(\theta(t))=M_{1}^{1} M_{3}^{1}, \\
& \lambda_{2}(\theta(t))=M_{1}^{1} M_{3}^{2}, \\
& \lambda_{3}(\theta(t))=M_{1}^{2} M_{3}^{1}, \\
& \lambda_{4}(\theta(t))=M_{1}^{2} M_{3}^{2},
\end{aligned}
$$

where the premise membership functions are as follows:

$$
\begin{aligned}
& M_{1}^{1}=\frac{x_{1}^{2}}{a^{2}}, \\
& M_{1}^{2}=1-M_{1}^{1}, \\
& M_{3}^{1}= \begin{cases}\frac{b \sin x_{3}-x_{3} \sin b}{x_{3}(b-\sin b)}, & x_{3} \neq 0 \\
1, & x_{3}=0,\end{cases} \\
& M_{3}^{2}=1-M_{3}^{1} .
\end{aligned}
$$

Suppose the initial condition $\varphi^{\mathrm{T}}(t)=\left[\begin{array}{llll}-1.2 & 0.5 & 0.7 & -0.6\end{array}\right]^{\mathrm{T}}$, $h=10$, and $\tau=0.27$; the state responses of the closedloop control system are shown in Figure 1. From Figure 1, the proposed state feedback controller can stabilize the original nonlinear system with input and state delays.

Example 3. Consider the following T-S fuzzy system with two rules [39]. The system matrix parameters are given as follows:

$$
A_{1}=\left[\begin{array}{ccc}
-a \frac{v \bar{t}}{L t_{0}} & 0 & 0 \\
a \frac{v \bar{t}}{L t_{0}} & 0 & 0 \\
-a \frac{v^{2} \bar{t}^{2}}{2 L t_{0}} & \frac{v \bar{t}}{t_{0}} & 0
\end{array}\right]
$$




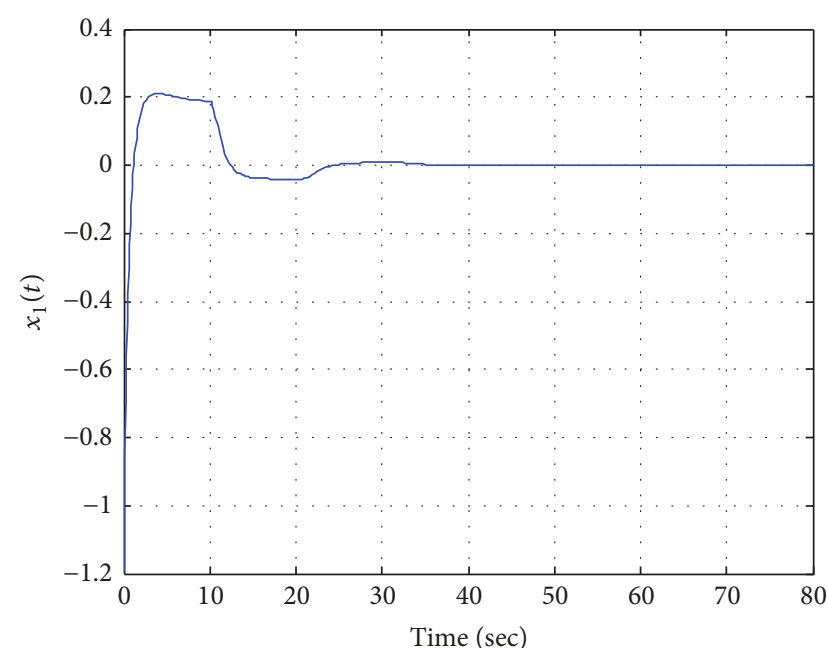

(a)

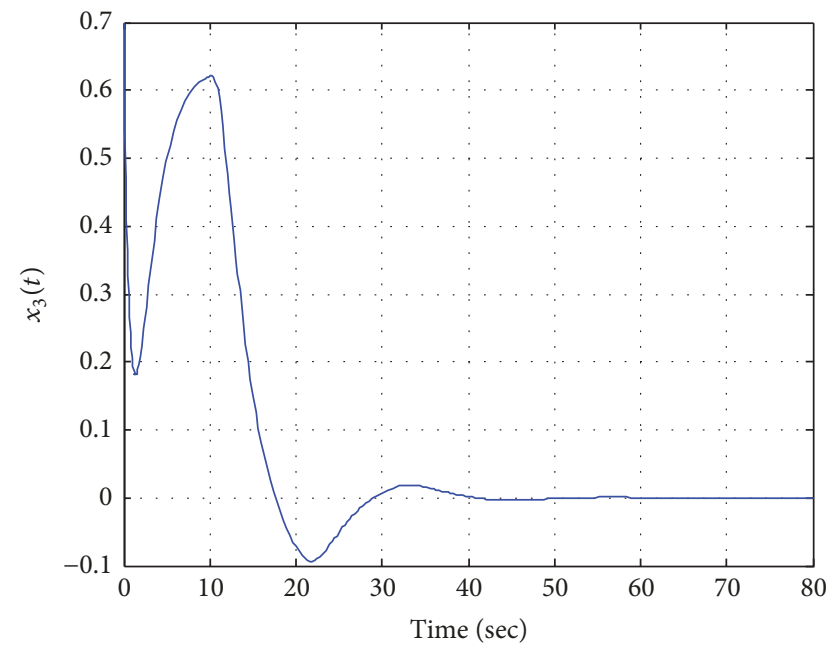

(c)

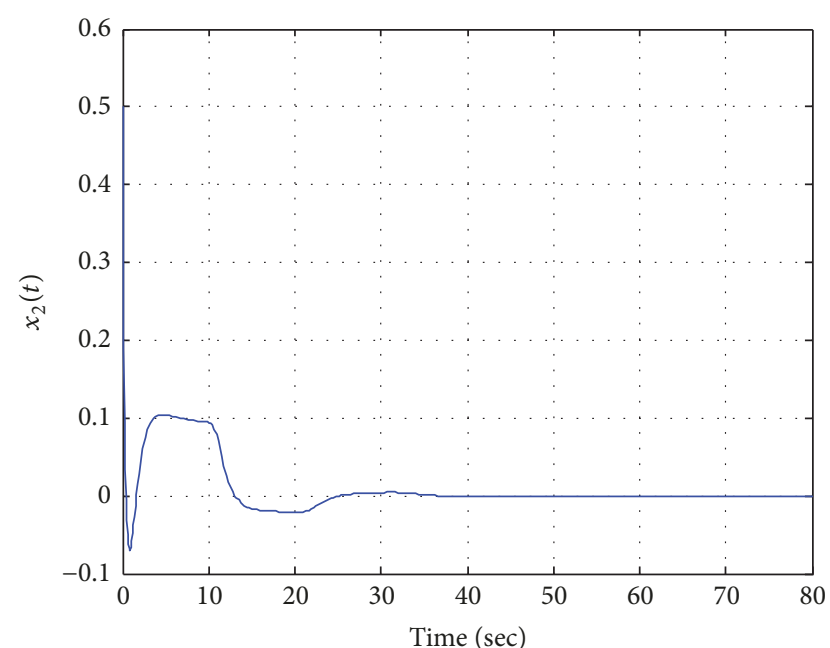

(b)

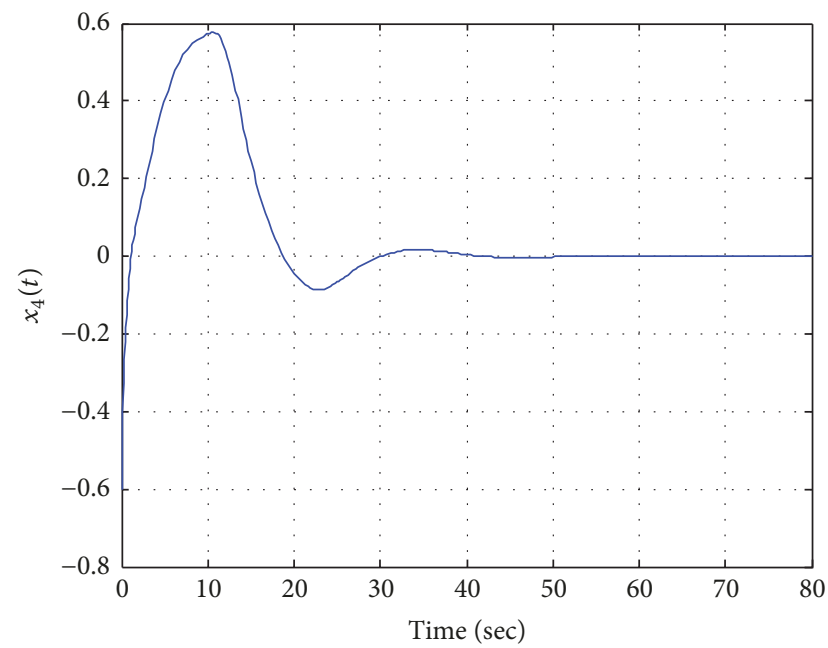

(d)

FIGURE 1: State responses of the closed-loop system for Example 2.

$$
\begin{aligned}
& A_{2}=\left[\begin{array}{ccc}
-a \frac{v \bar{t}}{L t_{0}} & 0 & 0 \\
a \frac{v \bar{t}}{L t_{0}} & 0 & 0 \\
-a d \frac{v^{2} \bar{t}^{2}}{2 L t_{0}} & \frac{d v \bar{t}}{t_{0}} & 0
\end{array}\right], \\
& B_{1}=\left[\begin{array}{c}
\frac{v \bar{t}}{l t_{0}} \\
0 \\
0
\end{array}\right], \\
& B_{2}=\left[\begin{array}{c}
\frac{v \bar{t}}{l t_{0}} \\
0 \\
0
\end{array}\right],
\end{aligned}
$$$$
A_{d 1}=\left[\begin{array}{ccc}
-(1-a) \frac{v \bar{t}}{L t_{0}} & 0 & 0 \\
(1-a) \frac{v \bar{t}}{L t_{0}} & 0 & 0 \\
(1-a) \frac{v^{2} \bar{t}^{2}}{2 L t_{0}} & 0 & 0
\end{array}\right] \text {, }
$$$$
A_{d 2}=\left[\begin{array}{ccc}
-(1-a) \frac{v \bar{t}}{L t_{0}} & 0 & 0 \\
(1-a) \frac{v \bar{t}}{L t_{0}} & 0 & 0 \\
(1-a) \frac{d v^{2} \bar{t}^{2}}{2 L t_{0}} & 0 & 0
\end{array}\right] \text {, }
$$

where $l=2.8, L=5.5, v=-1.0, \bar{t}=2.0, t_{0}=0.5, d=10 t_{0} / \pi$, $a=0.7$. 


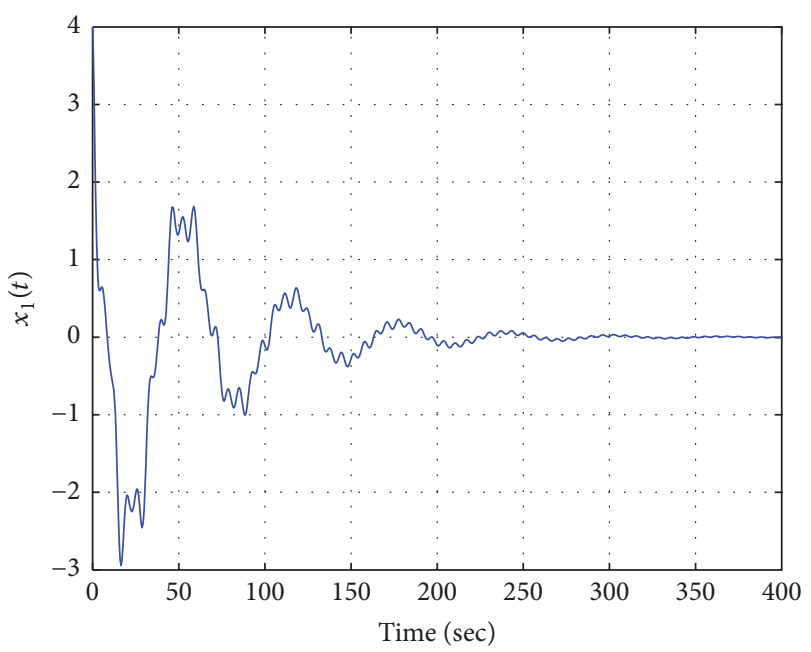

(a)

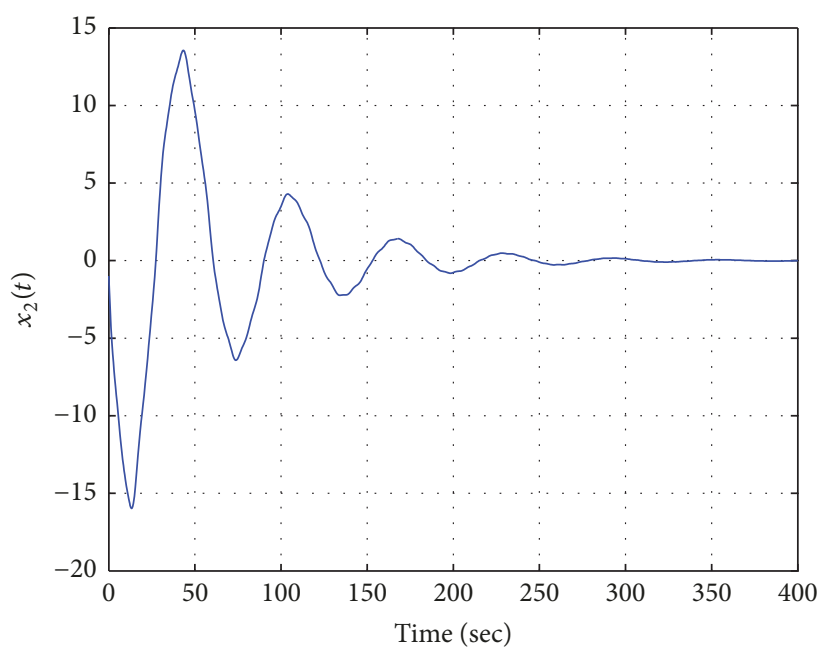

(b)

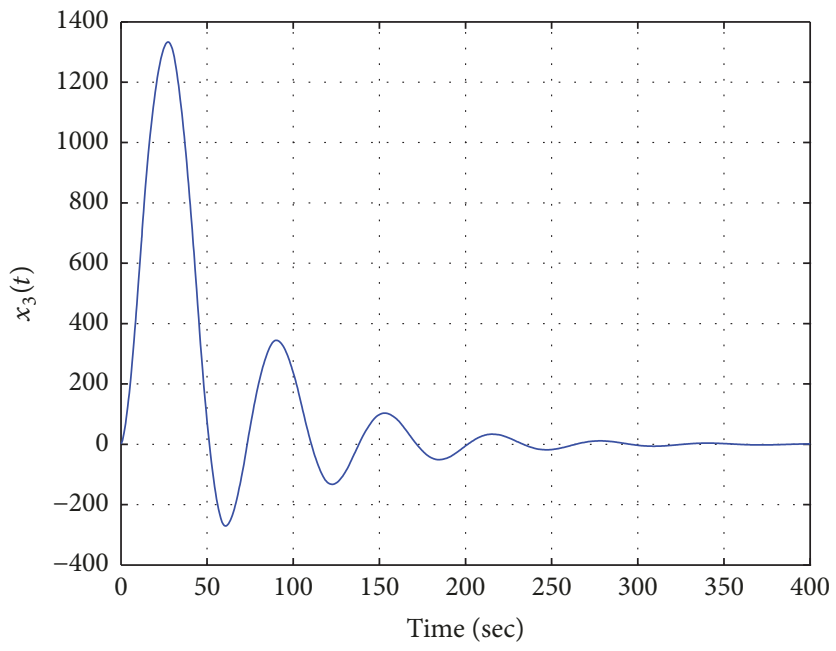

(c)

FiguRE 2: State responses of the closed-loop system for Example 3.

This example is taken from [39], which describes a T$S$ fuzzy system with state and input delay. In the following analysis, we give the comparison between our method and that proposed by [39]. By the method given in [39], the maximum allowable upper-bound of the time-delays is computed as $h=\tau=0.167$.

Then, setting parameters $a_{1}=1$ and $a_{2}=0.01$, according to Theorem 5 in our paper, the maximum allowable upperbounds of input and state delays are, respectively, computed as $h_{\max }=12.7$ and $\tau_{\max }=0.925$. Thus, the proposed method obtains less conservative results than that in [39].

Now, for simulation, we set the premise membership function as follows:

$$
\begin{aligned}
& \lambda_{1}(\theta(t))=\frac{1}{1+\exp \left(x_{1}+0.5\right)}, \\
& \lambda_{2}(\theta(t))=1-\lambda_{1}(\theta(t)) .
\end{aligned}
$$

Taking $h=12$ and $\tau=0.85$, and applying Theorem 5, we can obtain the feedback gain matrices as follows:

$$
\begin{aligned}
& K_{1}=\left[\begin{array}{lll}
0.6571 & -0.0459 & 0.0002
\end{array}\right], \\
& K_{2}=\left[\begin{array}{lll}
0.6576 & -0.0469 & 0.0002
\end{array}\right] .
\end{aligned}
$$

Figure 2 shows the state responses of the closed-loop system under the initial condition $\varphi^{\mathrm{T}}(t)=\left[\begin{array}{lll}4 & -1 & 2\end{array}\right]^{\mathrm{T}}$. From Figure 2, it can be seen that the proposed state feedback controller can stabilize the original T-S fuzzy system with input and state delays.

\section{Conclusion}

The stabilization problem of nonlinear systems with state and input delays is investigated via T-S fuzzy model in this paper. By choosing an appropriate Lyapunov functional, new delay-dependent stabilization criteria are established. Using Wirtinger-based double integral inequality, the proposed stabilization conditions are presented in the form of LMI. The largest allowable input and state delays calculated by the proposed conditions are obviously better than the existing 
results. Several examples are provided to demonstrate the effectiveness and superiority of the proposed method.

\section{Conflicts of Interest}

The authors declare that there are no conflicts of interest regarding the publication of this paper.

\section{Acknowledgments}

This work is supported by National Natural Science Foundation of China (61703291) and Applied Basic Research Program of Science and Technology Department of Sichuan Province, China (2016JY0085).

\section{References}

[1] T. M. Guerra, A. Sala, and K. Tanaka, "Fuzzy control turns 50: 10 years later," Fuzzy Sets and Systems, vol. 281, pp. 168-182, 2015.

[2] S. Tong, L. Zhang, and Y. Li, "Observed-based adaptive fuzzy decentralized tracking control for switched uncertain nonlinear large-scale systems with dead zones," IEEE Transactions on Systems, Man, and Cybernetics, vol. 46, no. 1, pp. 37-47, 2015.

[3] S. Tong, Y. Li, and S. Sui, "Adaptive fuzzy tracking control design for uncertain non-strict feedback nonlinear systems," IEEE Transactions on Fuzzy Systems, 2016.

[4] Y. Li, L. Liu, and G. Feng, "Robust adaptive output feedback control to a class of non-triangular stochastic nonlinear systems," Automatica, vol. 89, pp. 325-332, 2018.

[5] H. O. Wang, K. Tanaka, and M. F. Griffin, "An approach to fuzzy control of nonlinear systems: stability and design issues," IEEE Transactions on Fuzzy Systems, vol. 4, no. 1, pp. 14-23, 1996.

[6] H. K. Lam, F. H. F. Leung, and P. K. S. Tam, "Stable and robust fuzzy control for uncertain nonlinear systems," IEEE Transactions on Systems, Man, and Cybernetics: Systems, vol. 30, no. 6, pp. 825-840, 2000.

[7] R.-E. Precup, M. L. Tomescu, S. Preitl, E. M. Petriu, J. Fodor, and C. Pozna, "Stability analysis and design of a class of MIMO fuzzy control systems," Journal of Intelligent \& Fuzzy Systems: Applications in Engineering and Technology, vol. 25, no. 1, pp. 145-155, 2013.

[8] E. Fridman and L. Shaikhet, "Stabilization by using artificial delays: An LMI approach," Automatica, vol. 81, pp. 429-437, 2017.

[9] H. K. Lam and F. H. F. Leung, "Stability analysis of fuzzy control systems subject to uncertain grades of membership," IEEE Transactions on Systems, Man, and Cybernetics, Part B: Cybernetics, vol. 35, no. 6, pp. 1322-1325, 2005.

[10] X. Xie, D. Yue, and C. Peng, "Multi-instant observer design of discrete-time fuzzy systems: a ranking-based switching approach," IEEE Transactions on Fuzzy Systems, vol. 25, no. 5, pp. 1281-1292, 2017.

[11] X.-H. Chang, "Robust nonfragile Ho filtering of fuzzy systems with linear fractional parametric uncertainties," IEEE Transactions on Fuzzy Systems, vol. 20, no. 6, pp. 1001-1011, 2012.

[12] H. Li, Y. Gao, P. Shi, and H. K. Lam, "Observer-based fault detection for nonlinear systems with sensor fault and limited communication capacity," IEEE Transactions on Automatic Control, vol. 61, no. 9, pp. 2745-2751, 2016.
[13] T. Zhao and S. Dian, "Fuzzy dynamic output feedback Ho control for continuous-time T-S fuzzy systems under imperfect premise matching," ISA Transactions, vol. 70, pp. 248-259, 2017.

[14] X. Xie, D. Yang, and H. Ma, "Observer design of discretetime T-S fuzzy systems via multi-instant homogenous matrix polynomials," IEEE Transactions on Fuzzy Systems, vol. 22, no. 6, pp. 1714-1719, 2014.

[15] X. Xie, D. Yue, H. Zhang, and C. Peng, "Control synthesis of discrete-time T-S Fuzzy systems: reducing the conservatism whilst alleviating the computational burden," IEEE Transactions on Cybernetics, vol. 47, no. 9, pp. 2480-2491, 2017.

[16] X.-H. Chang and G.-H. Yang, "Relaxed stabilization conditions for continuous-time Takagi-Sugeno fuzzy control systems," Information Sciences, vol. 180, no. 17, pp. 3273-3287, 2010.

[17] X. P. Xie, D. Yue, and X. L. Zhu, "Further studies on control synthesis of discrete-time T-S fuzzy systems via useful matrix equalities," IEEE Transactions on Fuzzy Systems, vol. 22, no. 4, pp. 1026-1031, 2014.

[18] X.-P. Xie, Z.-W. Liu, and X.-L. Zhu, "An efficient approach for reducing the conservatism of LMI-based stability conditions for continuous-time T-S fuzzy systems," Fuzzy Sets and Systems, vol. 263, pp. 71-81, 2015.

[19] X. P. Xie, D. Yue, H. G. Zhang, and Y. S. Xue, "Control synthesis of discrete-time T-S fuzzy systems via a multi-instant homogenous polynomial approach," IEEE Transactions on Cybernetics, vol. 46, no. 3, pp. 630-640, 2016.

[20] S. Xu, J. Lam, B. Zhang, and Y. Zou, "A new result on the delay-dependent stability of discrete systems with time-varying delays," International Journal of Robust and Nonlinear Control, vol. 24, no. 16, pp. 2512-2521, 2014.

[21] X.-J. Xie, Z.-J. Li, and K. Zhang, "Semi-global output feedback control for nonlinear systems with uncertain time-delay and output function," International Journal of Robust and Nonlinear Control, vol. 27, no. 15, pp. 2549-2566, 2017.

[22] X. Xie and M. Jiang, "Output feedback stabilization of stochastic feedforward nonlinear time-delay systems with unknown output function," International Journal of Robust and Nonlinear Control, vol. 28, no. 1, pp. 266-280, 2018.

[23] B. Zhang, S. Xu, and Y. Zou, "Improved stability criterion and its applications in delayed controller design for discrete-time systems," Automatica, vol. 44, no. 11, pp. 2963-2967, 2008.

[24] T. Zhao, W. B. Liang, S. Y. Dian, J. Xiao, and Z. B. Wei, "Improved stability and stabilisation criteria for discrete time-delay systems via a novel double summation inequality," IET Control Theory and Applications, vol. 12, no. 3, pp. 327-337, 2018.

[25] A. Seuret and F. Gouaisbaut, "Wirtinger-based integral inequality: application to time-delay systems," Automatica, vol. 49, no. 9, pp. 2860-2866, 2013.

[26] M. Park, O. Kwon, J. H. Park, S. Lee, and E. Cha, "Stability of time-delay systems via Wirtinger-based double integral inequality," Automatica, vol. 55, pp. 204-208, 2015.

[27] W. Xiang, "Parameter-memorized Lyapunov functions for discrete-time systems with time-varying parametric uncertainties," Automatica, vol. 87, pp. 450-454, 2018.

[28] T. Zhao and S. Dian, "State feedback control for interval type-2 fuzzy systems with time-varying delay and unreliable communication links," IEEE Transactions on Fuzzy Systems, no. 99, 2017.

[29] T. Zhao and S. Dian, "Delay-dependent stabilization of discretetime interval type-2 T-S fuzzy systems with time-varying delay," Journal of The Franklin Institute, vol. 354, no. 3, pp. 1542-1567, 2017. 
[30] H.-N. Wu and H.-X. Li, "New approach to delay-dependent stability analysis and stabilization for continuous-time fuzzy systems with time-varying delay," IEEE Transactions on Fuzzy Systems, vol. 15, no. 3, pp. 482-493, 2007.

[31] J. Yoneyama, "New delay-dependent approach to robust stability and stabilization for Takagi-Sugeno fuzzy time-delay systems," Fuzzy Sets and Systems, vol. 158, no. 20, pp. 2225-2237, 2007.

[32] E. Tian and C. Peng, "Delay-dependent stability analysis and synthesis of uncertain T-S fuzzy systems with time-varying delay," Fuzzy Sets and Systems, vol. 157, no. 4, pp. 544-559, 2006.

[33] C. Peng, Y.-C. Tian, and E. Tian, "Improved delay-dependent robust stabilization conditions of uncertain T-S fuzzy systems with time-varying delay," Fuzzy Sets and Systems, vol. 159, no. 20, pp. 2713-2729, 2008.

[34] H. J. Gao, X. M. Liu, and J. Lam, "Stability analysis and stabilization for discrete-time fuzzy systems with time-varying delay," IEEE Transactions on Systems, Man, and Cybernetics, Part B: Cybernetics, vol. 39, no. 2, pp. 306-317, 2009.

[35] L. Wu, X. Su, P. Shi, and J. Qiu, "A new approach to stability analysis and stabilization of discrete-time T-S fuzzy timevarying delay systems," IEEE Transactions on Systems, Man, and Cybernetics, Part B: Cybernetics, vol. 41, no. 1, pp. 273-286, 2011.

[36] X. Su, P. Shi, L. Wu, and Y. Song, "A novel control design on discrete-time takagi-sugeno fuzzy systems with time-varying delays," IEEE Transactions on Fuzzy Systems, vol. 21, no. 4, pp. 655-671, 2013.

[37] L. Wu, X. Yang, and H. Lam, "Dissipativity analysis and synthesis for discrete-time T-S fuzzy stochastic systems with time-varying delay," IEEE Transactions on Fuzzy Systems, vol. 22, no. 2, pp. 380-394, 2014.

[38] C. Lin, Q.-G. Wang, and T. H. Lee, "Delay-dependent LMI conditions for stability and stabilization of T-S fuzzy systems with bounded time-delay," Fuzzy Sets and Systems, vol. 157, no. 9, pp. 1229-1247, 2006.

[39] B. Chen, X. Liu, S. Tong, and C. Lin, "Guaranteed cost control of T-S fuzzy systems with state and input delays," Fuzzy Sets and Systems, vol. 158, no. 20, pp. 2251-2267, 2007.

[40] B. Chen, X. Liu, C. Lin, and K. Liu, "Robust Ho control of Takagi-Sugeno fuzzy systems with state and input time delays," Fuzzy Sets and Systems, vol. 160, no. 4, pp. 403-422, 2009.

[41] X.-H. Chang, Robust Output Feedback H-Infinity Control And Filtering for Uncertain Linear Systems, Springer Science and Business, 2014.

[42] C. Z. Gong and S. Y. Li, "Design of controller for a class of uncertain T-S fuzzy model with time-varying input time delay," Journal of Civil Aviation University of China, vol. 24, no. 4, pp. 48-51, 2006.

[43] C.-H. Fang, Y.-S. Liu, S.-W. Kau, L. Hong, and C.-H. Lee, "A new LMI-based approach to relaxed quadratic stabilization of T-S fuzzy control systems," IEEE Transactions on Fuzzy Systems, vol. 14, no. 3, pp. 386-397, 2006. 


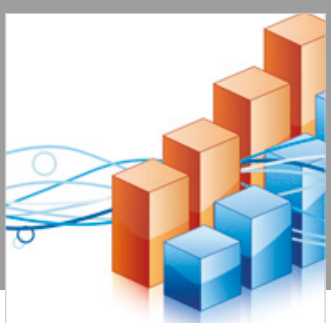

Advances in

Operations Research

\section{-n-m}
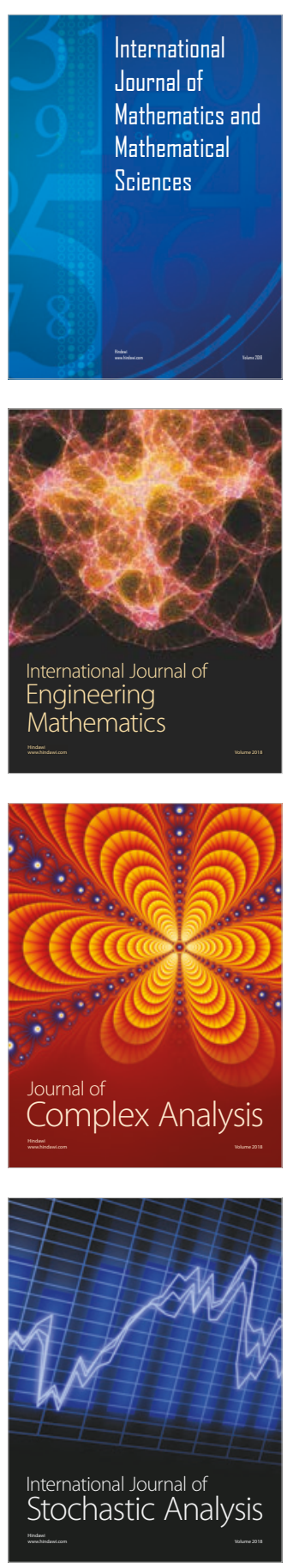
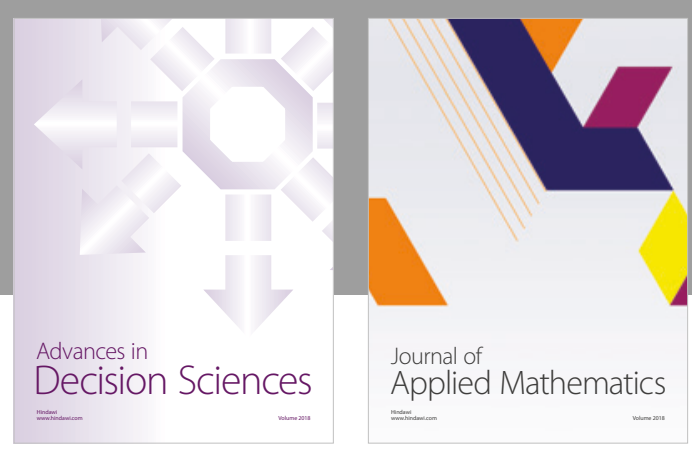

Journal of

Applied Mathematics
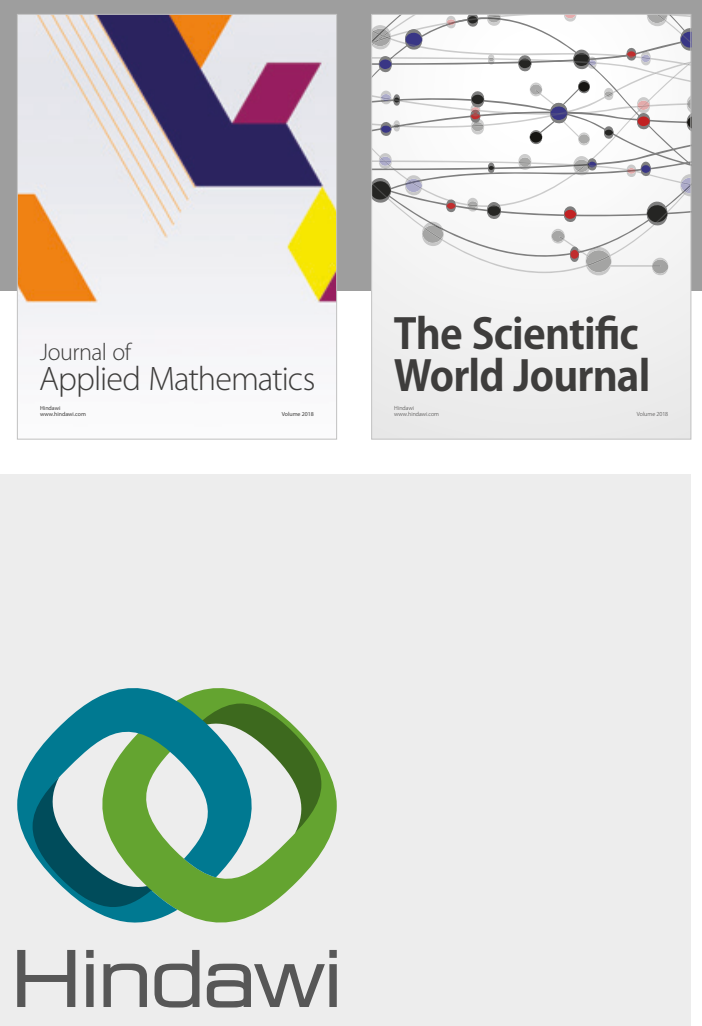

Submit your manuscripts at

www.hindawi.com

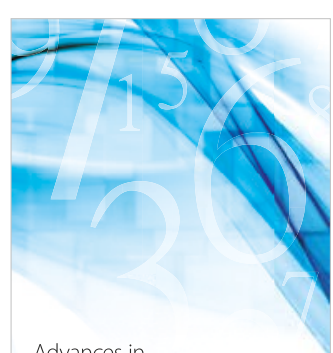

Advances in
Numerical Analysis
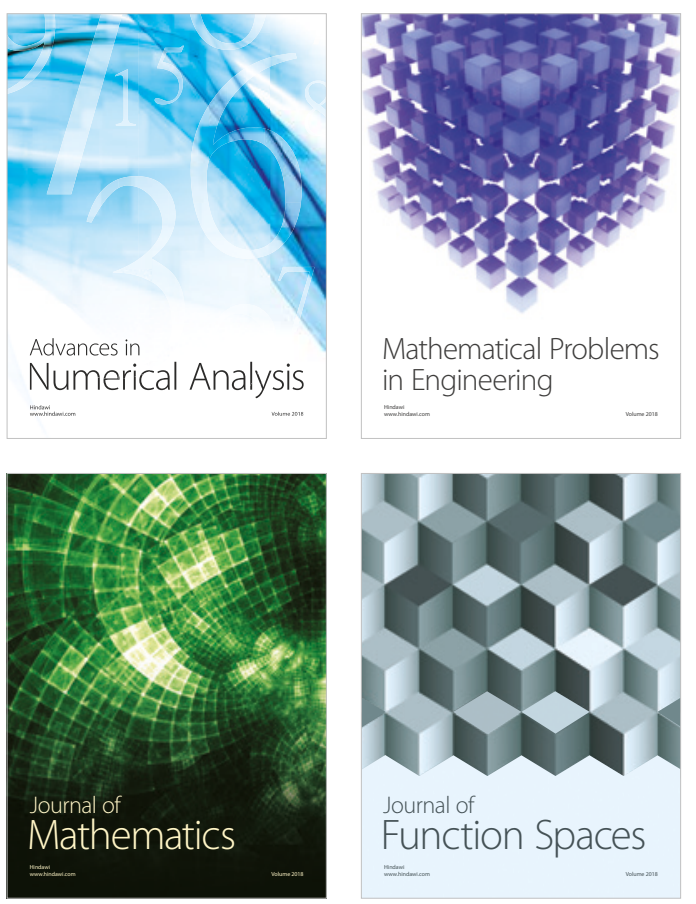

Mathematical Problems in Engineering

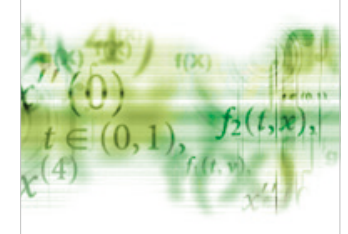

International Journal of

Differential Equations

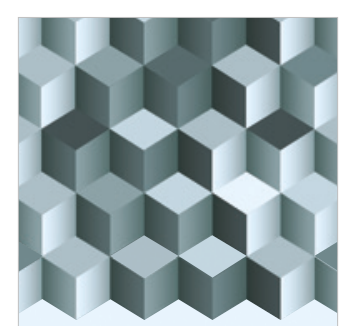

Journal of

Function Spaces

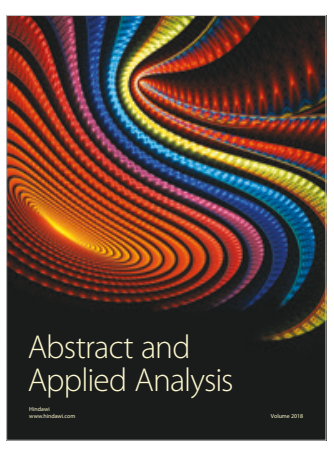

The Scientific

World Journal

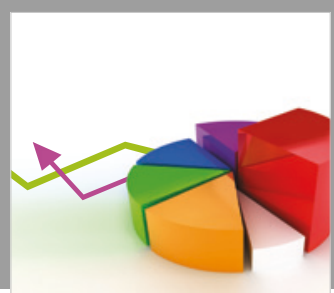

Journal of

Probability and Statistics
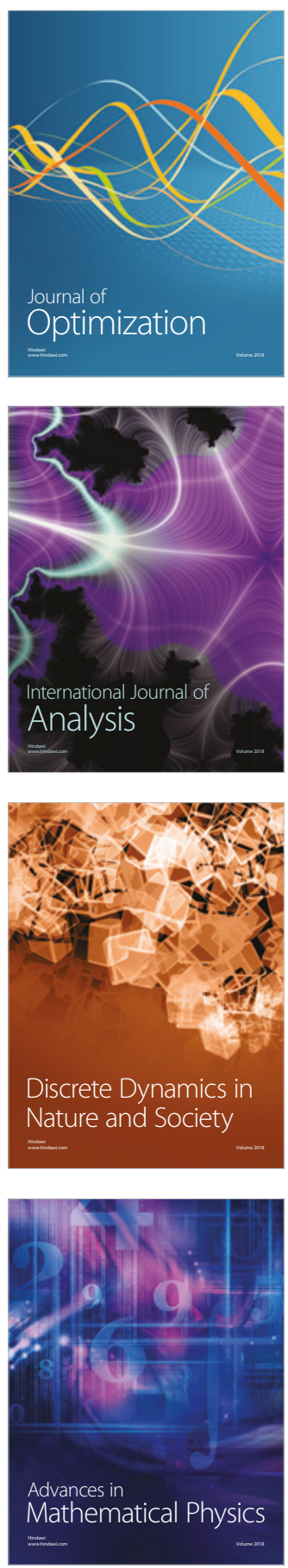